\title{
Data Integration in Poplar: 'Omics Layers and Integration Strategies
}

\author{
Deborah Weighill ${ }^{1,2 \dagger}$, Timothy J. Tschaplinski1,2, Gerald A. Tuskan ${ }^{2}$ and Daniel Jacobson ${ }^{1,2 *}$ \\ ${ }^{1}$ The Bredesen Center for Interdisciplinary Research and Graduate Education, University of Tennessee, Knoxville, Knoxville, \\ TN, United States, ${ }^{2}$ Biosciences Division, Oak Ridge National Laboratory, Oak Ridge, TN, United States
}

\section{OPEN ACCESS}

Edited by:

Josselin Noirel,

Conservatoire National des Arts et Métiers (CNAM),

France

Reviewed by: Jennifer Dick,

University of Sheffield,

United Kingdom

Guanglong Jiang,

Indiana University Bloomington,

United States

*Correspondence:

Daniel Jacobson

jacobsonda@ornl.gov

tPresent Address:

Deborah Weighill,

Department of Biostatistics, Harvard T.H. Chan School of Public Health,

Harvard University, Boston, MA,

United States

Specialty section:

This article was submitted to

Systems Biology,

a section of the journal

Frontiers in Genetics

Received: 23 March 2019

Accepted: 20 August 2019

Published: 25 September 2019

Citation:

Weighill D, Tschaplinski TJ,

Tuskan GA and Jacobson D (2019)

Data Integration in Poplar: 'Omics

Layers and Integration Strategies.

Front. Genet. 10:874.

doi: 10.3389/fgene.2019.00874
Populus trichocarpa is an important biofuel feedstock that has been the target of extensive research and is emerging as a model organism for plants, especially woody perennials. This research has generated several large 'omics datasets. However, only few studies in Populus have attempted to integrate various data types. This review will summarize various 'omics data layers, focusing on their application in Populus species. Subsequently, network and signal processing techniques for the integration and analysis of these data types will be discussed, with particular reference to examples in Populus.

Keywords: data integration, Populus, multi-omic data, networks, signal processing, wavelet transform

\section{INTRODUCTION}

Poplar species (Populus sp.) are promising sources of cellulosic biomass for biofuels because of their fast growth rate, high cellulose content, and moderate lignin content (Sannigrahi et al., 2010). Ragauskas et al. (2006) outline areas of research needed "to increase the impact, efficiency, and sustainability of biorefinery facilities," such as research into modifying plants to enhance favorable traits, including altered cell wall structure leading to increased sugar release, as well as resilience to biotic and abiotic stresses. One particular research target in Populus is the decrease/alteration of the lignin content of cell walls. The Populus genus contains a considerable amount of variation, is estimated to contain approximately 30 different species (Taylor, 2002), and is considered a model species for trees and woody species. Major Populus species and their characteristics can be found in the review by Taylor (2002).

There is an increasing movement towards integrating multiple layers of 'omics data in a systems biology approach to understand gene-phenotype relationships and assist in plant breeding programs [see Ingvarsson et al. (2016), Weckwerth (2011), and Valledor et al. (2018) for reviews]. Increasing systems biology knowledge through data integration in Populus species represents an important step in the development of Populus as a model system, as well as an efficient feedstock for biofuels through selective breeding programs and accelerated domestication (Tuskan, 2007). This review will discuss different sources of 'omics data layers with a particular focus on those previously applied in P. trichocarpa or other Populus species. We will also briefly mention 'omics layers that have not yet been applied in Populus species, but would be useful tools to consider to extend the systems biology knowledge of Populus species. Subsequently, we will review network and signal processing approaches to representing, analyzing, and integrating multiple 'omics data layers, again providing examples in Populus species where possible. 


\section{SOURCES OF 'OMICS DATA LAYERS}

\section{Overview}

Different 'omics layers each provide information on a different aspect of a complex biological system (Table 1). Below, we discuss different 'omics layers and the information they can provide about the system. We also present examples each of the 'omics data layers in the literature, focusing on examples in Populus species where available.

\section{Genomics}

\section{Genome and Annotation}

The genome sequence of Black Cottonwood, Populus trichocarpa (Torr. \& Gray) was released in 2006 (Tuskan et al., 2006). This genome, a single female genome "Nisqually-1," was the first tree to have its complete genome sequenced, and it became a model system for studies on woody perennial plants (Wullschleger et al., 2012; Jansson and Douglas, 2007). The P. trichocarpa genome consists of 19 chromosomes, with chromosome 19 predicted to be evolving into a sex chromosome (Yin et al., 2008). Analysis of homologous regions of the genome showed evidence for several whole-genome duplication events; the most recent being the Salicoid duplication event, which is contained within the family Salicaceae, the next termed the Eurosid duplication shared among Eurosids, and an ancient duplication event shared by all land plants (Tuskan et al., 2006).

Since initial sequencing, the genome assembly has gone through several revisions and is now in its fourth version. Furthermore, a genome-wide association study (GWAS) population of $\sim 1,000$ natural accessions from the United States and Canada was propagated in multiple common gardens and resequenced, providing a rich resource for studies of the variation in natural $P$. trichocarpa populations as well as GWASs (Tuskan et al., 2011; Slavov et al., 2012; Evans et al., 2014).

The genome sequence is available on Phytozome (Goodstein et al., 2012), and the genome along with gene and functional annotation such as Gene Ontology (GO) terms and PFams can

TABLE 1 | 'Omics data layers.

\begin{tabular}{ll}
\hline 'Omics Data layer & Information gained \\
\hline Genomics & $\begin{array}{l}\text { Primary DNA sequence, gene annotations, transposable } \\
\text { elements, repetitive sequences, genome variants } \\
\text { Transcriptomics }\end{array}$ \\
& $\begin{array}{l}\text { Gene expression, mRNA abundances, gene } \\
\text { of organism (cell, tissue) to different conditions at the } \\
\text { mRNA level }\end{array}$ \\
Metabolomics & $\begin{array}{l}\text { Metabolite abundances, response of organism to } \\
\text { different conditions at the metabolite level }\end{array}$ \\
Proteomics & Protein abundances, post-translational modifications, \\
& response of organism to different conditions at the \\
protein level & Associations between genomic variants and phenotypes \\
in a population, potential pleiotropic/epistatic \\
relationships \\
Epigenetic features such as DNA methylation, chromatin \\
Epigenomics
\end{tabular}

be viewed and interacted with using the JBrowse (Skinner et al., 2009) plugin on Phytozome (https://phytozome.jgi.doe.gov/pz/ portal.html).

This genomic data layer provides a base on which many other data sources can be layered, including various annotations and features of the genomic sequences as well as other data layers downstream in the central dogma of molecular biology.

\section{Genomic Variants}

Different individuals in a population can accumulate variation in their genome, such as single-nucleotide polymorphisms (SNPs) involving a nucleotide change at a single position, insertions/ deletions of a single nucleotide or larger pieces of DNA, copy number variations (CNVs) of DNA segments, or translocations (the movement of a section DNA from one location to another) (Abel and Duncavage, 2013).

There are two major approaches to calling SNPs in a given sample in a relatively high-throughput manner, namely, a genotyping SNP array and SNP calling from next-generation sequencing (NGS) data. A genotyping SNP array involves hybridizing extracted DNA to an array containing probes with known SNPs (LaFramboise, 2009) and is thus limited by the SNPs chosen to appear on the array. For example, the $P$. trichocarpa genotyping array is based on 34,131 SNPs located near/within around 3,500 selected candidate genes (Geraldes et al., 2013). SNP calling through NGS involves whole-genome shotgun sequencing of all individuals, aligning of all reads to a common reference genome, and then calling variants (Nielsen et al., 2011) using software such as GATK (McKenna et al., 2010). The advantage of SNP calling from NGS data is that one is not limited by the set of SNPs available on an array. A larger number of SNPs can be detected, and the discovery of new SNPs is possible. SNP genotyping arrays are also not able to detect other classes of genome variants such as translocations and inversions (LaFramboise, 2009).

A population of $\sim 1,000$ natural $P$. trichocarpa accessions have been clonally propagated in four common gardens (Tuskan et al., 2011) and resequenced in order to provide NGS data for SNP calling. Several studies have been published, making use of SNPs called across parts of this population (Slavov et al., 2012; Evans et al., 2014; McKown et al., 2014). Slavov et al. (2012) performed a study involving SNPs called from resequenced genomes of 16 of the genotypes within this P. trichocarpa population. PCA analysis of SNP genotypes revealed clear separation based on the geographic origin of the genotypes and linkage disequilibrium was reported to decay to $r^{2} \leq 0.2$ within $3-6 \mathrm{~kb}$. It is important to note that this is based on the resequencing of only 16 genotypes. A set of $\sim 28$ million bi-allelic SNPs called across 882 genotypes from this population have been publicly released and are available online from DOI 10.13139/OLCF/1411410.

\section{Genotype-Phenotype Associations}

Phenotypes are often complex traits, in that they are influenced or controlled by a great number of genes (Solovieff et al., 2013). GWASs attempt to associate the presence/ absence of SNPs with these complex traits (Visscher et al., 
2012; Solovieff et al., 2013). This involves genotyping a large sample of individuals of a population, measuring phenotypes across these individuals and statistically determining the association between the presence/absence of the genotyped markers or SNPs and the phenotypes across the population (Korte and Farlow, 2013). A general concern when conducting GWASs is that individuals within a population that are genetically related can share both causal alleles, which impact the phenotype (Visscher et al., 2012), and non-causal alleles (Korte and Farlow, 2013). These causal and non-causal alleles could be located nearby to each other on the chromosome and could thus be in linkage disequilibrium (LD) - alleles that are correlated across a population and thus co-inherited (FlintGarcia et al., 2003). This LD between causal and non-causal alleles across related individuals could result in non-causal alleles being correlated with a phenotype when they have no actual effect on the phenotype.

GWAS analyses generally require that the individuals in the population are unrelated. However, some level of population structure due to shared ancestors can cause spurious associations between genotype and phenotype, and accounting for population structure is thus important in order to remove variance that is due solely to the relatedness of individuals (for a useful review, see Astle and Balding et al., 2009). It is thus important to account for population structure in association models. However, there is the possibility of masking true associations that happen to correlate with population structure because they are local adaptations of clades to local environments.

An important element of GWAS studies is the issue of multiple hypothesis correction, as a GWAS typically involves the calculation of thousands or millions of statistical tests. An exhaustive review of different GWAS approaches and multiple hypothesis correction is beyond the scope of this review; however, we discuss these topics briefly in the Supplementary Text 1, and we refer the readers to useful articles on these topics (Noble 2009; Johnson et al., 2010; Bush and Moore, 2012; Korte and Farlow, 2013; Fadista et al., 2016; Visscher et al., 2017).

Several studies involving GWAS analyses in P. trichocarpa have been published. McKown et al. (2014) genotyped 448 individuals from the $P$. trichocarpa GWAS population using an SNP array containing 34,000 SNPs and performed GWAS on 40 different traits measured in the population. These traits included biomass phenotypes such as height, volume, and height:diameter ratio; ecophysiological traits such as leaf shape, chlorophyll content, and carbon:nitrogen ratio; and phenology traits such as bud set, growth period, and leaf drop. A set of 1118 significant GWAS associations were identified involving 410 unique SNPs, $78 \%$ of which occurred in non-coding regions and $28 \%$ occurred in coding regions. This resulted in 275 genes having significant trait associations, many of which were transcription factors or regulators of some kind. A subset of 42 of the 275 genes exhibited multiple GWAS associations with traits in different trait categories, exhibiting potential pleiotropy.

Evans et al. (2014) used whole-genome sequencing of 544 individuals from the $P$. trichocarpa GWAS population, and subsequent variant calling identified 17,902,740 SNPs. They found that 1) nucleotide diversity was twice as high in intergenic space than in genic space, 2) diversity was even lower in coding space, and 3) a large proportion of the SNPs had a minor allele frequency $(\mathrm{MAF}) \leq 0.01$ and were thus considered rare alleles. Metrics of natural selection, such as $F_{S T}$, were used to identify candidate regions under strong selection and suggest that this could be driven by climate.

Tuskan et al. (2018) tested callus induction in 280 genotypes from within the P. trichocarpa GWAS population and performed a GWAS analysis to identify SNPs potentially affecting callus formation. Eight genes potentially associated with callus formation were identified. Combining GWAS results with co-expression information allowed for a putative regulatory network for callus formation to be constructed.

In a recent study by Liu et al. (2018), 64 individuals from a fullsib family from a cross between $P$. deltoides and $P$. euramericana were genotyped using real-time PCR. Phenotypes used were stem heights and diameters over 24 years. Both a standard GWAS and distance correlation sure independence screening (DC-SIS) association tests are performed. DC-SIS is an association method that allows for a multi-dimensional phenotype (diameter measurements over time) as opposed to a single phenotype measurement.

\section{Repetitive and Transposable Elements}

Transposable elements (TEs) are segments of DNA that are mobile, in the sense that they can move from one genomic location to another. Type I elements, or retrotransposons, require an RNA intermediate, which is then reverse-transcribed into the genome at a different location (Slotkin and Martienssen, 2007; Wicker et al., 2007). This is thus a "copy and paste" mechanism. Type II TEs are called DNA transposons and involve the excision of the DNA TE and subsequent integration elsewhere. This can thus be described as a "cut and paste" mechanism (Slotkin and Martienssen, 2007; Wicker et al., 2007). Many TEs are no longer active because mutations have inhibited their ability to transpose. However, some TEs are silenced by the host. This can include mechanisms such as silencing by RNAi or though DNA/histone methylation (Slotkin and Martienssen, 2007).

Different TEs show preference for insertion at different locations in the genome, and thus exhibit very different distributions across the genome (Bennetzen and Wang, 2014). TEs have large impacts on genome characteristics and evolution (Klein and O'Neill, 2018). Firstly, they have a significant impact on genome size, comprising a large part of many plant genomes (Bennetzen and Wang, 2014), ranging from 10\% of the genome of Medicago truncatula, $42 \%$ of $P$. trichocarpa, and $80 \%$ of Pinus taeda (loblolly pine) (Kejnovsky et al., 2012). Unequal homologous recombination can also result from the presence of multiple TEs of a given family. This can cause various genome rearrangements including duplications, inversions, deletions, and translocations (Gaut et al., 2007; Bennetzen and Wang, 2014). TEs that insert into gene regions can cause the gene to become non-functional. In addition, TEs that insert near genes can impact the expression pattern of the genes, especially since some TEs contain regulatory sequences (Wicker et al., 2007; Bennetzen and Wang, 2014). Application of stress to an organism has been shown to activate TEs, leading to the hypothesis that 
TEs create variability in the genome that could be useful under times of stress (Capy et al., 2000).

Since the genome release, several investigations of repeats and TEs have been performed in P. trichocarpa. Soon after the release of the P. trichocarpa genome, Zhou and Xu (2009) annotated repeat sequences in the genome and made them publicly available in a database called RepPop. However, this database no longer appears to be available. Cossu et al. (2012) identified LTR repeats in $P$. trichocarpa and investigated their distribution across the genome, finding Gypsy LTRs to be enriched in putative centromeric regions. Soon after, Natali et al. (2015) surveyed LTR retrotransposons in an updated version of the $P$. trichocarpa genome. Vining et al. (2012) investigated the number of repeats and genes that were methylated vs non-methylated in $P$. trichocarpa, and found that methylated retroelements, LTRs, hAT elements, CACTA elements, and certain LINEs were overrepresented when compared to their un-methylated versions. It was also found that the methylation patterns of TEs differed significantly across tissues (Vining et al., 2012). Usai et al. (2017) performed an investigation into the repetitive DNA content of seven different Populus species, including P. deltoides, P. nigra, P. tremula, $P$. tremuloides, $P$. balsamifera, $P$. simonii, and $P$. trichocarpa. LTR repeats were the dominant repeat type across all species, although the total repeat content varied from $33.8 \%$ in P. nigra to $46.5 \%$ in $P$. tremuloides.

In a recent study by Mascagni et al. (2018), insertion ages of LTR TEs were determined in P. trichocarpa by comparing the sequences of the $3^{\prime}$ and $5^{\prime}$ ends of LTRs. This provides an indication of the time since insertion because at the time of insertion, the $3^{\prime}$ and $5^{\prime}$ LTRs are identical, and subsequently accumulate mutations independently after insertion. Insertion time was also determined by comparing the sequences of paralogous RTs from the same lineages. The two methods provided conflicting results, with the LTR comparison method suggesting that Gypsy TEs were older than Copia TEs, whereas the RT comparison method did not find a significant difference in the age of these classes. Yi et al. (2018) recently published a database (SPTEdb) of TEs in $P$. trichocarpa, P. euphratica, and Salix suchowensis. This database provides TE annotation for these organisms using multiple TE identification methods and presents these in a database format as well as a JBrowse interface.

\section{Transcriptomics}

Transcriptomic analysis involves the measuring of the expression levels of messenger RNA. Various study designs have been implemented in $P$. trichocarpa to investigate a variety of properties of the cellular system. Several studies have focused on the response of the Populus transcriptome, or a subset of the transcriptome, to drought stress. The study by Shuai et al. (2013) used RNA-Seq to identify microRNAs responsive to drought stress, and subsequently, Shuai et al. (2014) performed RNASeq on control and drought leaf samples of $P$. trichocarpa to identify long intergenic non-coding RNAs (lincRNAs) that were responsive to drought stress. Tang et al. (2015) used RNA-Seq to identify genes differentially expressed between well-watered and water-limited samples, and several differentially expressed genes and functions were identified. Genes related to energy metabolism and growth (cell division and tissue expansion) were significantly downregulated, and Potri.013G093600, a homolog of an Arabidopsis thaliana vacuolar pyrophosphatase (AVP1) was significantly upregulated. This gene had been previously found to improve drought and salt tolerance in several plants. Another transcriptomic drought study used Affymetrix microarrays for expression measurements of $P$. tremula $\times P$. alba roots for six time points under drought stress. Differential expression and network analysis identified two interesting genes (PtaJAZ3 and PtaRAP2.6), which, when overexpressed under drought conditions, increased root growth (Dash et al., 2018).

Other transcriptomic studies in Populus have focused on variation in gene expression across tissues or across a population. In the study by Quesada et al. (2008), gene expression levels in $P$. trichocarpa were measured across five different tissues (roots, young leaves, mature leaves, nodes, and internodes) using NimbleGen microarrays. Genes with tissue-specific gene expression were identified, with stem samples having the highest number of tissue-specific genes. GO enrichment was used to determine the enriched functions of organ-specific genes. The expression of $P$. trichocarpa genes across organs was also compared to the expression of their A. thaliana orthologs across equivalent tissues, and the authors concluded that, while there were some similarities between expression patterns across these two species, significant diversification in gene expression regulation has occurred between orthologs. Shi et al. (2009) used quantitative real-time PCR (qPCR) to determine the expression level of 95 genes in the phenylpropanoid pathway in xylem, leaf, shoot, and phloem tissues, in order to determine the abundance and tissue specificity of genes potentially involved in monolignol biosynthesis. Bao et al. (2013) performed RNASeq of xylem tissue from $20 \mathrm{P}$. trichocarpa individuals from different populations, identified a set of genes expressed in xylem across all individuals, and found several instances of alternative splicing, particularly in cell wall-related genes and that these alternative splicing events differed significantly across individuals.

An increasingly common study design is the construction of a gene expression atlas for a species, which involves determining the expression level of every gene in the genome in various different tissues and/or conditions. Gene expression atlas studies have been performed in various plant species (see, for example, Table 2), and several expression atlas datasets are available on Phytozome (phytozome.jgi.doe.gov). The P. trichocarpa RNA-Seq gene expression atlas consists of genome-wide gene expression measurements across several different samples of tissue and condition combinations, including root, root tip, stem, node, internode, bud, leaf, and flower tissues. Root and stem tissues included several samples varied by nitrogen source. Bud, leaf, and male and female flowers included several samples of different stages of maturity. Gene expression values for 40 of these samples are currently publicly available in PhytoMine on the Phytozome web interface (Goodstein et al., 2012; https://phytozome.jgi.doe. gov/index.html). To our knowledge, this is the largest RNA-Seq expression study performed in Populus. 
TABLE 2 | Examples of gene expression atlas studies in plants.

\begin{tabular}{|c|c|c|c|}
\hline Species & Reference & Samples & Method \\
\hline Arabidopsis thaliana & Schmid et al. (2005) & 79 samples from various tissues and developmental stages & Affymetrix GeneChip \\
\hline Sorghum bicolor & McCormick et al. (2018) & $\begin{array}{l}47 \text { combinations of tissues (roots, leaves, stems, panicles) and } \\
\text { developmental stages (juvenile, vegetative, reproductive) }\end{array}$ & RNASeq \\
\hline Glycine max & Severin et al. (2010) & 14 tissues from different developmental stages & RNASeq \\
\hline Lotus japonicus & Verdier et al. (2013) & 237 samples of 8 tissues across various conditions & Affymetrix GeneChip \\
\hline Medicago truncatula & Benedito et al. (2008) & 18 samples from tissues across different developmental stages & Affymetrix GeneChip \\
\hline Barley & Druka et al. (2006) & 15 tissues identified from eight developmental stages & Affymetrix GeneChip \\
\hline Rice & Wang et al. (2010) & $\begin{array}{l}31 \text { tissues spanning life cycle of rice plant for } 2 \text { rice varieties, } 8 \\
\text { samples from stages in the tissue culture process }\end{array}$ & Affymetrix GeneChip \\
\hline $\begin{array}{l}\text { Panicum virgatum L } \\
\text { (Switchgrass) }\end{array}$ & Zhang et al. (2013) & $\begin{array}{l}\text { Tissues (roots, shoots, and panicle) and developmental stages (leaf } \\
\text { development, stem elongation and reproduction) }\end{array}$ & ESTs \\
\hline Vitis vinifera & Fasoli et al. (2012) & 54 samples from tissues spanning different developmental stages & NimbleGen microarray and RNASeq \\
\hline
\end{tabular}

\section{Metabolomics}

Metabolomics studies involve measuring the quantities of metabolites within a sample. While targeted metabolomics studies aim to only measure and identify a select few metabolites within a sample (for instance using standards), untargeted metabolomics involves the measuring as many metabolites as possible within a sample (Patti et al., 2012). Identification of metabolites in untargeted metabolomics studies is much more challenging than that of targeted metabolomics studies. While the candidate identities of many metabolite peaks can be determined through database matching or manual inspection of mass spectra with the necessary expertise, many metabolites will remain unidentified or partially identified.

Several targeted and untargeted metabolomics studies have been performed in Populus. In a study by Morreel et al. (2006), metabolite levels of 15 flavonoids were measured using highperformance liquid chromatography (HPLC), and subsequently mQTL (metabolite quantitative trait loci) based on amplified fragment length polymorphisms (AFLPs) was used to identify potential genes involved in rate-limiting steps of flavonoid biosynthesis. Kaling et al. (2015) performed untargeted metabolomics on UV-B treated vs. control $P$. alba $\times P$. tremula plants using Fourier transform ion cyclotron resonance mass spectrometry (FT-ICR-MS). This allowed for the investigation of the effect of UV radiation on the metabolome. Tuskan et al. (2012) performed gas chromatography-mass spectrometry (GC/MS) analysis of 16 individual trees in $P$. deltoides and $P$. nigra, and showed gender-specific accumulations of metabolites in floral buds. In Hamanishi et al. (2015), transcriptomic and metabolomic data of six $P$. balsamifera were collected using Affymetrix microarrays and GC/MS, respectively, to investigate the response of the metabolome and transcriptome to drought stress. Tschaplinski et al. (2014) used GC/MS-based metabolomics on samples of $P$. trichocarpa and $P$. deltoides roots colonized with Laccaria bicolor as well as control samples to investigate the different metabolic responses to colonization. One interesting result was that increased levels of defense-related compounds were found in the incompatible host, $P$. deltoides, whereas some defense compounds were significantly lower in the compatible host, $P$. trichocarpa. A recent study by Veach et al. (2018) investigated the effects on the metabolome of $P$. deltoides when downregulating PdKOR1, a glycosyl hydrolase gene involved in cellulose biosynthesis. GC/MS analysis of root tissue from PdKOR1 RNAi lines vs. control lines showed that caffeic acid derivatives, metabolites involved in fatty acid metabolism as well as salicylates and flavonoids were upregulated in RNAi lines when compared to control lines Veach et al. (2018). Additionally, Tschaplinski et al. (2019) reported the differential foliar metabolomic responses of $P$. deltoides responding to acute vs. chronic drought, with the former inducing the largest osmotic adjustment $(1.42 \times)$, with the greatest accumulations in the large, complex higher-order salicylate conjugates, and hydroxycinnamic acid conjugates of salicin; the populosides were particularly elevated.

A GWAS using SNPs from 917 P. trichocarpa accessions as well as GC/MS-based metabolomics and RNA-Seq-based gene expression measurement identified hydroxycinnamoylCoA:shikimate hydroxycinnamoyl transferase 2 (PtHCT2) as a gene that is significantly associated with the levels of 3-O-caffeoylquinic acid, and also identified transcription factors that regulate this gene (Zhang et al., 2018).

\section{Proteomics}

Proteomics involves identifying and quantifying the levels of the protein component of cells within a sample. This is an important layer of data to consider, as it allows for the investigation of the cellular components that participate directly in metabolic pathways, cell structure, and development (Chen and Harmon, 2006). The relationship between protein levels and transcript levels varies depending on the scenario. For example, as reviewed by Liu et al. (2016b), while "at steady state, mRNA levels primarily explain protein levels," this relationship can change when measuring transcript/protein levels after a state transition, as there is an expected delay between mRNA and protein synthesis (Liu et al., 2016b). The relationship between mRNA and protein levels is thus complicated, and we refer the readers to Liu et al. (2016b) for a detailed review on the dependencies between these two 'omics layers. It is important to note that separate identification and quantification of proteins is important to fully understand this 'omics layer, including the set of post-translational modifications undetectable through other 'omics layers.

Several proteomics studies have been performed in Populus investigating changes in the proteome due to different 
developmental stages or conditions. There is a particular abundance of studies investigating the response of the Populus proteome to drought stress. Zhang et al. (2010) investigated the sex-related differences proteomic response to drought stress in $P$. cathayana and found significant sex-dependent responses to drought stress, particularly in chloroplast-related processes such as the Calvin cycle, electron transport chains, and chloroplast components. They also found that the growth rates of male trees were less affected by drought than females, and that chloroplasts were less damaged by drought in males than females (Zhang et al., 2010). Durand et al. (2011) performed a proteomic study to investigate the different drought responses of different tissues in Populus tremula L. $\times$ P. alba $L$., illustrating how some tissues are affected sooner by drought than others. Abraham et al. (2018) investigated the proteome of $P$. deltoides in response to different types of drought stress. Two separate drought treatments were used: a cyclic drought treatment and a prolonged, "slow-drying" water deficit. Differentially abundant proteins were determined for each of the two drought treatments, and, interestingly, these sets of differentially abundant overlapped by only around $10 \%$. This study illustrated diversity in responses to different types of water deficit stress.

Several studies have also investigated proteome profiles at different stages of development in Populus species (Liu et al., 2015; Obudulu et al., 2016). Proteomics is thus an important data layer that can provide information about cellular function and responses that could not be gained by other 'omics layers. As the "end point" of the central dogma, proteins are the biomolecules that provide a large part of the functional capacity of a cell, and are a crucial aspect of understanding the functioning of the system.

\section{Epigenomics \\ DNA Methylation}

Epigenetics involves the study of additions of chemical groups to chromatin (either the DNA or histones) that do not change the underlying DNA sequence. These modifications consist of histone methylation (Liu et al., 2010), histone acetylation (Lusser et al., 2001), and DNA methylation (Finnegan et al., 1998). Histone methylation occurs on lysine and arginine residues in histones and can have a silencing or activating effect on gene expression, depending on which lysine residue is methylated (Liu et al., 2010). Histone acetylation involves the addition of an acetyl group to the $\epsilon$ amino group of lysine residues in the $\mathrm{N}$-terminal tails of histones that protrude from the histone octamer complex (Lusser et al., 2001). While histones are usually positively charged, and DNA is negatively charged, acetylation can neutralize the positive charge of the histones, resulting in a weaker association between the DNA and the histone complex. This can allow for greater access for transcription factors to the DNA and can thus impact gene expression (Lusser et al., 2001). DNA methylation involves the addition of a methyl group to cytosine residues (Law and Jacobsen, 2010). This is known to have a gene-silencing affect. DNA methylation in plants occurs mostly in repetitive DNA and TEs. This is thought to be a protective mechanism to silence transposons. DNA methylation is also found within the transcribed regions of genes in plants (Suzuki and Bird, 2008). This gene body methylation does not have a silencing effect like promotor methylation does, but appears to lead to stable gene expression across many tissues (Zilberman et al., 2007; Suzuki and Bird, 2008). Epigenetic modifications can be inherited from parents or occur as a result of a stress response (Chinnusamy and Zhu, 2009; Lämke and Bäurle, 2017).

Two major whole-genome sequencing-based approaches for determining DNA methylation across a genome are methyl-DNA immunoprecipitation (MeDIP) followed by sequencing (MeDIPSeq) (Jacinto et al., 2008) or treatment of DNA with bisulfite followed by sequencing (Frommer et al., 1992; Krueger et al., 2012). MeDIP-Seq involves shearing of DNA into small fragments of 300-600 bp and subsequent immunoprecipitation of methylated DNA using an antibody raised against 5-methylcytidine. The resulting immunoprecipitated fragments are sequenced, and mapping of the reads to the reference genome reveals the regions of the genome that contain methylated cytosines. It is important to note that the resolution of the methylation results cannot exceed the fragment size. In bisulfite sequencing, DNA is pretreated with sodium bisulfite, which converts un-methylated cytosine residues to uracil residues, while methylated cytosine residues remain unchanged. Subsequent sequencing provides single-base resolution of methylated cytosines (Frommer et al., 1992; Krueger et al., 2012). See published papers of Laird (2010) and Bock (2012) for useful reviews on DNA methylation analysis.

Vining et al. (2012) investigated DNA cytosine methylation in seven different tissues in P. trichocarpa, including bud, male catkin, female catkin, leaf, root, xylem, and phloem. DNA methylation was determined using MeDIP-Seq followed by mapping of reads to the $P$. trichocarpa reference genome. Reads mapped most frequently to intergenic regions and repeat sequences, although promotor methylation and gene body methylation were observed. Variation in methylation across tissues was observed at certain chromosomal locations. A surprising result was that gene body methylation appeared to be a stronger repressor of transcription than promotor methylation (Vining et al., 2012). Slavov et al. (2012) made use of the methylation data generated by Vining et al. (2012) in investigating the correlates of recombination in the $P$. trichocarpa genome and found that DNA within recombination hotspots were significantly less methylated than non-hotspots.

A follow-up study by Vining et al. (2013) used MeDIP-Seq to examine methylation levels in another three tissues, focusing on regeneration and de-differentiation tissue types, namely, internode stem from propagated explants, callus, and internodes from regenerated plants. The MeDIP-Seq reads for the 10 different $P$. trichocarpa tissues from these two studies have been mapped to the version 3.2 genome assembly and are available on Phytozome (Goodstein et al., 2012).

A stress response methylome study was performed in $P$. trichocarpa in which DNA methylation was measured in drought stress and control plants using bisulfite sequencing (Liang et al., 2014). The number of methylated cytosines increased significantly under drought stress and the genes differentially methylated in drought stress vs control plants were enriched for regulatory GO terms. This study also performed the first investigation of alternative splicing in $P$. trichocarpa and identified multiple forms of alternative splicing. An interesting finding was also that all fusion genes identified were methylated (Liang et al., 2014). 
Lafon-Placette et al. (2013) investigated the component of the $P$. trichocarpa methylome in open chromatin by isolating chromatin sensitive to DNase I and performing MeDIP-Seq on the resulting DNA. Extensive gene body methylation was found, more so than was originally reported for $P$. trichocarpa.

The two studies by Vining et al. $(2012,2013)$ provide the best available methylation dataset to use as an association network layer in P. trichocarpa as it covers the broadest range of sample types.

\section{AtAC-Seq}

The assay of transposase-accessible chromatin (ATAC-Seq) uses a transposase to insert sequencing adaptors into accessible regions of chromatin (the areas in between nucleosomes) (Buenrostro et al., 2013, Buenrostro et al., 2015). The resulting fragments are then PCR-amplified and sequenced. This results in nucleotide resolution of open chromatin. There were challenges in applying this method to plant cells due to contaminating DNA from chloroplasts and mitochondria because chloroplast and mitochondria genomes are very accessible to the transposase and thus lower the efficiency of the technique. Lu et al. (2016) developed a technique, fluorescence-activated nuclei sorting (FANS)-ATAC-Seq, which involves sorting of nuclei using flow cytometry prior to ATAC-Seq analysis. Bajic et al. (2018) describe protocols for the isolation of plant nuclei from different cell types for further analysis using ATAC-Seq. A recent study by Maher et al. (2018) applied ATAC-Seq to A. thaliana, M. truncatula, Solanum lycopersicum (tomato), and Oryza sativa (rice). An interesting finding was that in all four species, most open chromatin sites were in non-transcribed regions.

ATAC-Seq is a relatively new technology, and, to date, no study has been published on the application of ATAC-Seq in P. trichocarpa.

\section{DAP-Seq}

DNA affinity purification sequencing (DAP-seq) is a technique used to determine transcription factor binding sites (O'Malley et al., 2016). This technique involves coupling a particular transcription factor of interest to affinity beads. Fragmented genomic DNA is eluted over the beads, retaining only DNA fragments that bind to the transcription factor. Subsequently, the retained fragments are sequenced. The first study describing this technique demonstrated its use in identifying the Arabidopsis "cistrome"-the binding location/motifs of 1,812 transcription factors (O'Malley et al., 2016). The DAP-seq protocol was published by Bartlett et al. (2017).

To date, no DAP-seq study has been performed in $P$. trichocarpa. This would be an incredibly valuable data layer to investigate the transcription factor regulatory network of P. trichocarpa.

\section{DATA INTEGRATION}

\section{Multi-Omic Studies and Data Integration}

The current era has an extensive suite of technologies capable of measuring and characterizing several aspects of a cellular system, such as NGS technologies for genomics, transcriptomics, and epigenomics as well as metabolomics and other phenotypes.
An untargeted approach is often favored over a targeted approach as this attempts to capture information about the entire system and understand the organism as a whole. In the review by Weckwerth (2011), it is highlighted that the next step in understanding complex systems will involve the integration of these different data layers. An important and challenging task that data integration can help solve is the identification of new candidate genes involved in complex phenotypes (Hassani-Pak and Rawlings, 2017; Valledor et al., 2018), which can then be validated using genetic/molecular biology tools. It is particularly difficult to generate hypotheses that suggest the mechanism of a gene's effect on a particular phenotype. Prioritizing candidate genes and hypothesizing the mechanism of the effect requires multiple data types, such as gene-phenotype associations, expression/co-expression information, knowledge from literature, annotation information, protein-protein/proteinDNA interactions, and epigenetic modifications, to name a few (Hassani-Pak and Rawlings, 2017). This presents a challenge because of the heterogeneous nature of these data types, and the fact that they are often distributed across different databases and represented as different structures (Hassani-Pak and Rawlings, 2017). There is thus an increasing value in databases that integrate various layers of data from various sources (Hu et al., 2018), for example, Knetminer (Hassani-Pak et al., 2016; Hassani-Pak, 2017) and String (Mering et al., 2003; Szklarczyk et al., 2010; Fukushima and Kusano, 2014; Szklarczyk et al., 2017).

Data integration requires that the various data layers be coerced into a uniform data structure. The data collected from various techniques can each be represented as a matrix/table of samples and variables, as illustrated in the review by Weckwerth (2011). Once represented as a matrix, there are various data structures/analysis approaches that can be used to integrate and analyze the data. This can range from multivariate analysis such as Orthogonal Projections to Latent Structures (OPLS) (Bylesjö et al., 2007) to networks (Krishnan et al., 2016; Hassani-Pak and Rawlings, 2017) and signal processing, such as that seen in the study by Spencer et al. (2006).

There are two main data integration strategies that have been applied in poplar, namely, network-based data integration and signal-based integration. While there are many useful databases that present poplar gene networks or different sources of 'omics data [see, for example, "GFDP: the gene family database in poplar" (Wang et al., 2018a) and also the useful review by Lee et al. (2015)], we will focus below on strategies that have computationally integrated several different 'omics data layers. This section describes the theory behind integration strategies and data structures/approaches applied in poplar, such as networks and signal processing. Examples of the various 'omics layers in these data structures are then presented. Thereafter, examples in which these data structures/methods are used in the analysis of multiple biological data types are discussed, focusing on examples in Populus species.

\section{Networks}

\section{Network Theory}

Networks are useful mathematical structures that represent a system in terms of its components, and pairwise interactions 
between the components (Barabasi and Oltvai, 2004). The field of Network Theory has its origins in Graph Theory. Intuitively, a graph (or network) is a set of objects (nodes) connected by lines (edges) as shown in Figure 1A. In biological network applications, nodes represent a biological object of interest and edges represent associations/interactions/similarities between these biological objects.

A graph can be represented numerically as a matrix, namely, an Adjacency Matrix (Golumbic, 2004). The Adjacency Matrix associated with the small example graph in Figure 1A is shown in Figure 1B. Each edge $e_{i j}$ in a graph can be assigned a real number weight $w_{i j}$ that represents the strength of the relationship between the two nodes it connects. A weighted graph can be mathematically represented as a Weighted Adjacency Matrix. This matrix is constructed in a similar manner to the normal Adjacency Matrix.

A bipartite graph involves nodes that can be partitioned into two non-overlapping sets. Intuitively, this means that a bipartite graph (or a bipartite network) consists of two classes of nodes in which nodes of one class can only be connected to nodes of the other class. An example of a bipartite network is shown in Figure 1C, and its matrix representation is shown in Figure 1D. Mathematical definitions of networks and adjacency matrices can be found in Supplementary Text 2.

Networks are useful tools for modeling and analyzing complex biological systems by representing biological molecules/ components as nodes (e.g. genes, proteins or metabolites) and representing the relationships/interactions/similarities between them as edges (Barabasi and Oltvai, 2004). For example, networks can model co-expression relationships between genes, sequence similarity between genes, physical interactions between proteins, or correlations between metabolites. Bipartite networks are particularly useful when representing relationships between different types of biological objects/concepts. For example, Goh et al. (2007) use bipartite networks to represent the human "diseaseome," connecting human diseases to their associated genes. Also, Weighill et al. (2019a) used bipartite network representations of GWAS results in order to characterize potentially pleiotropic associations in P. trichocarpa. As discussed in Weighill et al. (2019a), bipartite networks are useful structures for the representation and visualization of high-dimensional data. One set of nodes in a bipartite network can represent the variables (axes) of a space, while the other set of nodes represents the points within that space (samples). This representation

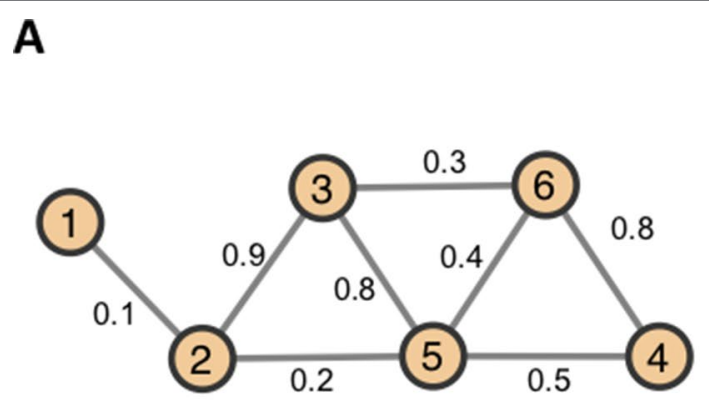

B

\begin{tabular}{|l|l|l|l|l|l|l|}
\hline & $\mathbf{1}$ & $\mathbf{2}$ & $\mathbf{3}$ & $\mathbf{4}$ & $\mathbf{5}$ & $\mathbf{6}$ \\
\hline $\mathbf{1}$ & 0 & 0.1 & 0 & 0 & 0 & 0 \\
\hline $\mathbf{2}$ & 0.1 & 0 & 0.9 & 0 & 0.2 & 0 \\
\hline $\mathbf{3}$ & 0 & 0.9 & 0 & 0 & 0.8 & 0.3 \\
\hline $\mathbf{4}$ & 0 & 0 & 0 & 0 & 0.5 & 0.8 \\
\hline $\mathbf{5}$ & 0 & 0.2 & 0.8 & 0.5 & 0 & 0.4 \\
\hline $\mathbf{6}$ & 0 & 0 & 0.3 & 0.8 & 0.4 & 0 \\
\hline
\end{tabular}

C

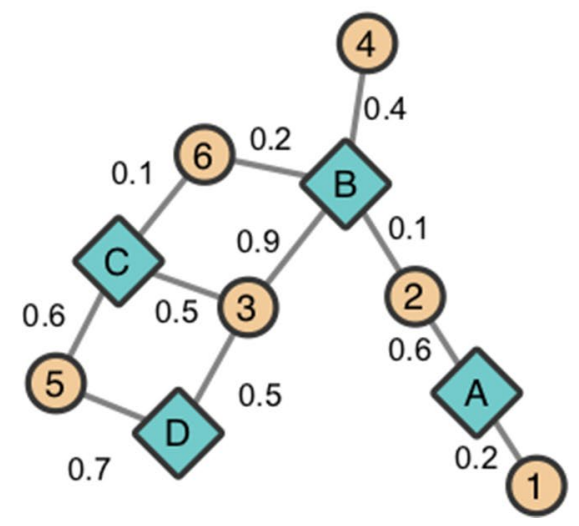

\begin{tabular}{|l|l|l|l|l|}
\hline & A & B & C & D \\
\hline $\mathbf{1}$ & 0.2 & 0 & 0 & 0 \\
\hline $\mathbf{2}$ & 0.6 & 0.1 & 0 & 0 \\
\hline 3 & 0 & 0.9 & 0.5 & 0.5 \\
\hline 4 & 0 & 0.4 & 0 & 0 \\
\hline 5 & 0 & 0 & 0.6 & 0.7 \\
\hline 6 & 0 & 0.2 & 0.1 & 0 \\
\hline
\end{tabular}

FIGURE 1 | Example networks. A small, standard undirected network represented (A) visually as a collection of nodes and edges and (B) as an adjacency matrix. A bipartite network represented (C) visually and (D) as an adjacency matrix. 
allows for high-dimensional datasets to be visualized in two dimensions as a network. Networks allow for biological datasets to be visualized in an intuitive manner and network visualization packages such as Cytoscape (Shannon et al., 2003) provide an interactive environment for network visualization. However, networks are not simply useful as a visualization tool. Networks provide a data structure that can be computed upon, allowing further analysis to be performed on a dataset represented as a network. Examples of such analysis methods include networkbased clustering algorithms such as Markov Clustering (MCL) (Enright et al., 2002) and Weighted Gene Co-expression Network Analysis (WGCNA) (Zhang and Horvath, 2005) that cluster the nodes of a network into groups based on the topology of the underlying network. Datasets represented as networks are also very easily merged with each other. This feature makes networks a useful tool for combining information from different data sources to create an integrated and holistic environment for data interpretation.

\section{GWAS Networks}

Network approaches have been applied to GWAS analyses in order to interpret or further analyze the resulting lists of SNPs and $p$ values. These often involve mapping the resulting SNPs associated with phenotypes to their respective genes, and then projecting these genes into protein-protein interaction networks (Akula et al., 2011) or co-expression networks (Farber, 2013) in order to identify other putative causal genes, or to form sets or subnetworks of genes putatively affecting the same phenotype (Leiserson et al., 2013).

The results of a GWAS can be viewed as a bipartite network where the set of nodes can be partitioned into a set of SNPs and a set of measured phenotypes, and the edges connect SNP nodes to phenotype nodes they are significantly associated with. SNPs can be connected to multiple phenotypes, and phenotypes can be connected to multiple SNPs. A toy example of such a network can be seen in Figure 1C. Teal, diamond-shaped nodes represent measured phenotypes A-D and orange, circular nodes represent SNPs 1-6. Each edge represents GWAS associations between SNPs and phenotypes. This representation of GWAS results has been used to estimate pleiotropy within a Human-Phenotype Network, calculated as the average degree of the gene nodes within a gene-phenotype bipartite network (Darabos et al., 2014). Another example can be seen in a study by Fagny et al. (2017) in which the results of an expression quantitative trait loci (eQTL) were represented as a bipartite network, connecting SNPs to genes if the expression level of the gene was significantly associated with the SNP (Fagny et al., 2017). Recently, Weighill et al. (2019a) presented a method for characterizing multiphenotype association signatures from GWAS results in order to investigate potentially pleiotropic interactions between genes and phenotypes. This method involves a decomposition relationship between three GWAS-derived bipartite networks, which allow for detailed pleiotropic signatures to be characterized, and, furthermore, allows for genes to be clustered based on the detailed topology of SNP-phenotype associations within the gene. This method was demonstrated on metabolomic GWAS results in $P$. trichocarpa and suggests applications of this method in identifying promising target genes of interest to modification or selective breeding (Weighill et al., 2019a).

\section{Co-Expression, Co-Methylation, and Correlation Networks}

Several of the 'omics data layers discussed in the section Sources of 'Omics Data Layers can be used to construct gene networks, such as gene co-expression networks and gene co-methylation networks. These networks require some quantity, such as gene expression, to be measured for every gene across multiple samples representing different conditions, tissues, or perturbations. A common way to construct gene association networks is to calculate the similarity between the profiles of all pairs of genes (Figure 2) and then apply a threshold [for examples, see Li et al. (2015) and Weighill and Jacobson (2017)]. The choice of similarity metric can have a large impact on the resulting network topology, as shown in a study by Weighill and Jacobson (2017).

Co-expression networks have been used for various applications, including gene function investigations, gene module and regulatory hub gene investigations, as well as comparative co-expression network analysis across different species

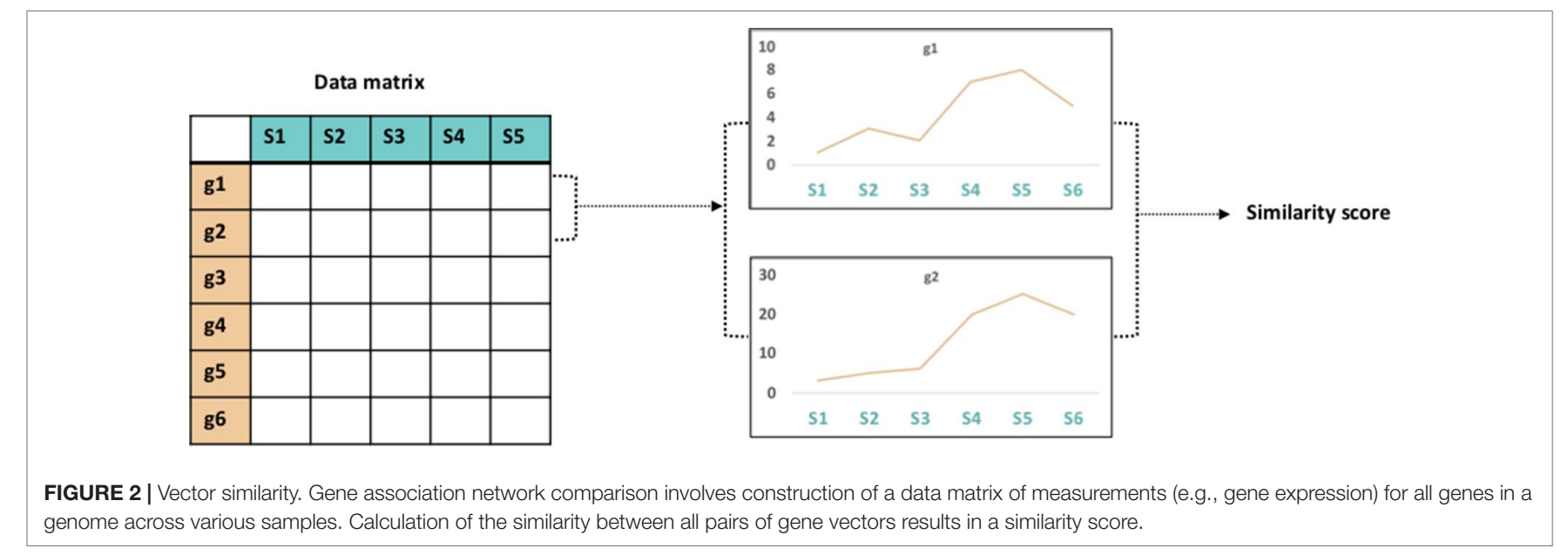


(Aoki et al., 2007; Li et al., 2015; Serin et al., 2016; Emamjomeh et al., 2017; Schaefer et al., 2017). Movahedi et al. (2012) described an approach for incorporating gene homology information in order to compare gene co-expression modules across plant species to identify clusters that are conserved across species. The overall functional impact of modules of sets of co-expressed genes can be investigated using enrichment of functional ontologies such as GO (Gene Ontology Consortium, 2004) and MapMan (Thimm et al., 2004) [see, for example, Emamjomeh et al. (2017)].

Horvath and Dong (2008) presented a useful set of network topology measures to characterize the structure of a co-expression network (or any network). These measures ranged from global network measures such as centralization, density, and heterogeneity to node-based metrics such as connectivity and the clustering coefficient. Yip and Horvath (2007) also developed a new network measure/transformation called Topological Overlap, which calculates the "connectedness" of two nodes based on direct connections as well as indirect connections via their neighbors. This provides an extra transformation that can be performed on a similarity network, which considers not only the similarity between expression profiles of two genes in question but also the expression profiles of their network neighbors, and thus can help address the problem arbitrary thresholds missing important edge connections. This Topological Overlap measure is an integral part of a popular gene co-expression pipeline called WGCNA developed by Langfelder and Horvath (2008). An extension of the Topological Overlap measure, called CrossNetwork Topological Overlap, was developed by Weighill and Jacobson (2017), which can be used to compare the similarity in the neighborhoods of a given node in two distinct networks.

Several studies in Populus species have involved co-expression networks, some focusing on co-expression networks as the main aspect of the investigation, and others using co-expression networks as a supplementary investigation surrounding the functions of a specific set of genes. Netotea et al. (2014) investigated differences in the genome-wide co-expression networks of $P$. trichocarpa, $O$. sativa, and A. thaliana constructed from publicly available expression data. It was found that while individual gene-gene co-expression relationships were different between the three species, overall neighborhoods of genes were significantly conserved across species. Another interesting finding was that orthologs with the most sequence similarity did not have the most similar expression pattern ["expressolog," as defined in Patel et al. (2012)].

An interesting co-expression study by Grönlund et al. (2009) constructed co-expression networks from 1024 publicly available microarray datasets for $P$. tremuloides by jack-knife re-sampling half of the number of samples 100 times, calculating the Pearson correlation between all pairs of genes in each jackknife re-sample, converting the Pearson correlations to distance metrics, and subsequently constructing 100 minimum spanning trees (MSTs) and merging the resulting networks. This approach of re-sampling allowed for the identification of rarer interactions between genes that would not have been identified through only looking at the dataset as a whole. Another whole genome co-expression study in Populus was performed by Ogata et al. (2009) in which 95 publicly available $P$. trichocarpa microarray expression datasets were used to construct a co-expression network and extracted co-expression modules, which were released in a publicly available database.

Several studies of specific genes in Populus incorporated co-expression elements into their analysis. Tian et al. (2017) investigated the role of $P$. trichocarpa $\mathrm{Na}+/ \mathrm{H}+$ antiporters in stress responses, as well as potential functional divergences within the family of these NHX genes. Using a co-expression network from publicly available data on Phytozome, they showed divergence in the expression pattern of members of this family. Several studies in Populus performed WGCNA of genes responding to certain stresses/conditions, including control vs drought conditions (Xue et al., 2016) in P. tremula $\times$ alba, controls vs jasmonic acid, and salicylic acid treatments in a $P$. deltoides $\times P$. euramericana hybrid (Luo et al., 2019), and also a developmental gradient of stem tissue (Chao et al., 2019). In a characterization of DWARF14 genes in $P$. trichocarpa, co-expression networks showed divergent expression between the two DWARF14 (Zheng et al., 2016). In another recent study by Tuskan et al. (2018), genes having a GWAS association with callus formation were identified, and the co-expression patterns of these genes were investigated using a co-expression network constructed from the $P$. trichocarpa gene expression atlas, and identified interesting clusters of positive and negative co-expression relationships between these genes, showing a clear regulatory pattern. It is evident that co-expression networks are a well-developed and widely used data layer in various organisms including Populus.

Co-methylation networks are a newer approach looking at the similarity between the methylation patterns of genes, and a more limited number of studies using co-methylation networks were found. However, they are a valid and useful data layer that carries information not present in co-expression datasets.

In a study by van Eijk et al. (2012), methylation and gene expression data were collected for several human individuals to investigate the relationship between these two data layers. WGCNA was used to construct co-expression and co-methylation networks, and subsequently to identify co-expression and co-methylation modules. In general, co-expression and co-methylation modules had very few overlapping genes, although both co-expression and co-methylation modules showed significant functional enrichment for various GO terms. Linear regression was also used to identify relationships between methylation and expression across individuals in which both positive and negative relationships were identified (van Eijk et al., 2012). Various other co-methylation network analyses have been performed in human cancer investigations (Akulenko and Helms, 2013; Bartlett et al., 2014; Ha et al., 2015).

\section{SNP Correlation}

SNP correlation networks involve calculating the correlation/ co-occurrence between SNPs across a population, and can be converted to gene-gene networks by mapping SNPs to the genes in which they reside. The Custom Correlation Coefficient (CCC) is an allele-specific correlation metric proven to be useful in identifying sets of SNPs ("blocs") that can be tested against complex phenotypes to uncover combinatorial genetic associations that affect the phenotype (Climer et al., 2014a; Climer et al., 2014b). 
The edges in the SNP correlation network can also be interpreted as potential co-evolutionary relationships, particularly when the variants in question reside on different chromosomes. The CCC is defined for bi-allelic SNPs. For a given pair of sites $i$ and $j$, the CCC is calculated four times, once for each pair of alleles $x$ and $y$ between the two sites. The CCC between alleles $x$ and $y$ at positions $i$ and $j$, respectively, is defined as:

$$
\operatorname{CCC}_{i_{x} j_{y}}=\frac{9}{2} R_{i_{x} j_{y}}\left(1-\frac{1}{f_{i_{x}}}\right)\left(1-\frac{1}{f_{j_{y}}}\right)
$$

where $R_{i_{x} j_{y}}$ represents the relative co-occurrence of $x$ and $y$ at positions $i$ and $j, f_{i_{x}}$ represents the frequency of allele $x$ at position $i$, and $f_{j_{y}}$ represents the frequency of allele $y$ at position $j$ (Climer et al., 2014a; Climer et al., 2014b).

CCC has been used to investigate the genetic underpinnings of heart disease (Climer et al., 2014b), psoriasis (Climer et al., 2014a), and genes implicated in various other diseases (Climer et al., 2015). This metric was also applied in a study by Bryan et al. (2018) in P. trichocarpa. The positioning of the two DWARF14 paralogs in the $P$. trichocarpa SNP correlation network was investigated, indicating that they appeared to have different co-evolution partners, potentially indicating functional divergence (Bryan et al., 2018). The CCC metric has been ported to run on graphics processing units (GPUs) providing a significant increase in speed (Joubert et al., 2018; Joubert et al., 2019).

Kogelman and Kadarmideen (2014) constructed SNP correlation modules by calculating the Pearson correlation between pairs of variants across individuals followed by topological overlap clustering using WGCNA. This method was termed "WISH" (Weighted Interaction SNP Hub) and was considered an extension of WGCNA to genotype data. Later, in 2017, the developers of WGCNA published an extension of the method to construct SNP correlation networks from GWAS associations, termed "WSCNA" (Weighted SNP Correlation Network Analysis), which involves clustering SNPs based on beta coefficients from a GWAS analysis (Levine et al., 2017), and describe the use of these networks in calculating polygenic risk scores.

\section{Network-Based Data Integration}

A useful review by Gligorijević and Pržulj (2015) classifies network-based data integration into two categories, namely, homogeneous and heterogeneous integration. Homogeneous integration involves integrating networks with the same type of nodes, but different edge types, for example, a gene co-expression network and a gene interaction network. Heterogeneous data integration involves integrating networks with both different node types and edge types. These strategies for data integration are then subdivided into groups based on the stage at which data integration occurs. Early integration involves integration of the datasets, and a single model is built on a combined dataset. This appears similar to the definition of Concatenation-based integration as described by Ritchie et al. (2015). Late integration involves building separate models from each individual dataset and subsequently combines the information in the separate models. This is similar to Model-based integration as described by Ritchie et al. (2015). A third integration strategy described by Ritchie et al. (2015), transformation-based integration, involves transforming multiple datasets into an intermediate, common structure, such as a network, which are then merged before the constructing further models.

Two of the most exhaustive network-based data integration tools are String (Search Tool for the Retrieval of Interacting Genes) and KnetMiner, both of which are online, freely accessible resources. STRING is an online, publicly available database of protein interactions, incorporating various data types and data sources, including co-expression, co-occurrence, physical interactions, sequence homology, and associations from textmining (Mering et al., 2003; Szklarczyk et al., 2010; Szklarczyk et al., 2017). The user can search for genes and resulting network neighborhoods can also be clustered using K-means clustering and MCL (Enright et al., 2002). Protein 3D structure as well as functional enrichment information is also displayed. The STRING database can also be queried through the Cytoscape network visualization app (Shannon et al., 2003; Szklarczyk et al., 2017). Certain sets of publicly available data for P. trichocarpa are available in STRING. KnetMiner is a publicly available tool/database consisting of heterogeneous "knowledge" networks for 11 species, including $P$. trichocarpa, and includes layers of information of different types and sources represented as networks, such as GWAS data, sequence homology relationships, annotation information, metabolic pathways, protein interactions, and occurrence in scientific literature (Hassani-Pak et al., 2016; Hassani-Pak, 2017). KnetMiner allows the user to search not only for genes, but also for concepts, phenotypes, or pathways. A score (KNETscore) is then calculated to rank genes based on their relevance of the neighborhood to the search terms. KnetMiner provides useful network visualizations as well as a chromosomal view indicating the location on the chromosomes in which the genes occur and an "evidence view" indicating the number of nodes/concepts of different types in the neighborhood of the genes in question.

The Mergeomics $\mathrm{R}$ package and webserver allows one to integrate GWAS summary statistics with other biological pathways and gene networks, and perform enrichment analyses as well as Weighted Key Driver Analysis (Arneson et al., 2016). This involves identifying hub genes in a selected/uploaded gene network, and subsequently overlaying phenotype-associated genes from uploaded GWAS analyses, and reports key drivers for each of these genes (Arneson et al., 2016). Key drivers and their neighborhoods can then be visualized using Cytoscape Web.

Mizrachi et al. (2017) developed an interesting network-based data integration approach to combine pathway information from KEGG, eQTL associations, and gene expression data in Eucalyptus. The network-based integration approach involves constructing a gene interaction network based on information in KEGG as well as eQTL associations with biomass and wood traits. The adjacency matrix of this network is then multiplied with a gene expression matrix, which results in a "network-diffused gene expression" matrix. This adjusts gene expression values based on those of neighboring genes in the gene interaction 
network. These new gene expression profiles are then correlated with each trait to identify genes of relevance to wood properties and biomass (Mizrachi et al., 2017).

Walley et al. (2016) performed a study that compared the topologies of various gene association networks in Maize. A gene co-expression network, a protein-co-expression network, and a phosphoprotein co-expression network were constructed and clustered into modules using WGCNA, and the edge conservation between the networks was calculated using the Jaccard index, and found that $6.1 \%$ of the edges were shared between the protein co-expression and gene co-expression networks. Functional enrichment using MapMan (Thimm et al., 2004) terms was performed on modules of co-expressed genes/ proteins from the two networks, and similar enriched functions were found in both networks.

NetICS (Network-based Integration of Multi-omics Data) is a data integration strategy based on graph diffusion (Dimitrakopoulos et al., 2018). This method was developed by Dimitrakopoulos et al. (2018) in order to prioritize cancer genes. A directed gene interaction network was constructed from publicly available data that included multiple types of relationships, including phosphorylation, co-expression, activation, and inhibition. Aberrant genes (i.e., those found to be differentially impacted in a case/control experiment) are marked and network diffusion is used to predict "mediator genes" that link upstream "genetically aberrant" genes to downstream gene expression changes (Dimitrakopoulos et al., 2018). This approach successfully identified many known cancer genes.

Gutiérrez et al. (2007) investigated gene expression in $A$. thaliana under carbon and nitrogen treatments. A separate gene interaction network was constructed using publicly available protein-protein and protein-DNA interactions, as well as miRNA-RNA interactions and the Arabidopsis metabolic pathway. A subnetwork consisting of $\mathrm{C} / \mathrm{N}$ responsive genes and their neighbors in the multi-network was constructed. Clustering of this subnetwork revealed interesting regulatory subnetworks.

Bunyavanich et al. (2014) used a multi-omic networkbased approach to investigate allergic rhinitis. GWAS was performed on 5633 genotyped individuals, and gene expression was measured in 200 of these individuals. Gene co-expression network and modules were constructed using WGCNA. Co-expression modules that contained genes that harbored or were near to GWAS-associated genes were considered candidate modules associated with allergic rhinitis. Associations between SNPs and gene expression were determined (called "eSNPs"), which are SNPs within 1 $\mathrm{MB}$ of a gene, which is also associated with the expression of the gene. Modules enriched in eSNPs were also identified, and it was found that the candidate allergic rhinitis modules were enriched in eSNPs associated with allergic rhinitis, and mitochondrial pathways were identified as important components of allergic rhinitis using functional enrichment (Bunyavanich et al., 2014).

Calabrese et al. (2017) integrated GWAS and co-expression data in an investigation into genes affecting bone mineral density. Genes identified as associated with bone mineral density in a GWAS analysis were mapped onto a co-expression network, which was subsequently clustered into modules. Co-expression modules that were enriched for GWAS hits were then identified.

Liu et al. (2016a) constructed a "co-functional" gene network for P. trichocarpa, making use of multiple data sources, including genomics data, poplar gene expression data from microarray experiments, as well as various sources of annotation including PFAM, GO, KEGG pathways, MapMan annotations, and MetaCyc. The co-functional network is accessible through the PoplarGene webserver, which also contains tools for gene prioritization (Liu et al., 2016a).

Weighill et al. (2018) presented a "Lines of Evidence" (LOE) approach for integrating data and identifying new candidate genes involved in a function of interest. The LOE approach takes as input a set of anchor genes/phenotypes thought to be involved in a given function of interest based on annotation and literature/expert knowledge. Thereafter, a LOE score is calculated for every gene in the genome, quantifying its connectivity to the input anchor genes across various 'omics network layers. These scores then allow genes to be ranked based on how much evidence exists connecting them to a given function across several data layers. This method, demonstrated and applied in $P$. trichocarpa, integrated several layers of association networks, including a gene co-expression network, gene co-methylation network, gene co-evolution network, as well as two GWAS networks and identified new promising candidate genes potentially involved in lignin biosynthesis and regulation (Weighill et al., 2018). The association networks constructed in this study were also used to provide context to candidate genes identified in a multi-trait GWAS analysis in $P$. trichocarpa, using combinations of 14 morphological/ physiological traits to identify candidate genes involved in these traits (Chhetri et al., 2019).

There have thus been several efforts to integrate various data layers, sometimes for the goal of prioritizing candidate genes, and others for providing biological context for the interpretation of GWAS results.

\section{Signal Processing Data Representation}

In the previous section, we discussed the representation of biological data at network structures, which focuses on relationships between pairs of objects. Here, we discuss the representation of biological data as "signals" and subsequent analysis techniques.

A biological signal represents the response of a variable over some range of input values, which usually have some longitudinal feature, such as a response over increasing time, or a response over increasing distance. Classic examples of biological signals are feature density signals across chromosomes, such as SNP density, gene density, recombination density, and GC content, to name a few (Spencer et al., 2006; Paape et al., 2012; McCormick et al., 2018).

These signals have variation at different scales (i.e., are composed of multiple signals of different frequencies), and 
signal processing techniques can be used to extract frequency information. McCormick et al. (2018) who used the Fourier Transform to identify a prominent periodicity in SNP density, finding that SNP density peaked with a period of 3 base pairs downstream of coding sequence start sites, which was explained by the positions in the third "wobble" base being under lower selective pressure.

The Fourier transform represents a signal as a linear combination of sine and cosine waves. These are infinite waves and thus the Fourier transform provides no information as to which frequencies are observed at different locations in the signal. The wavelet transform is a newer signal processing technique that addresses this limitation (Leavey et al., 2003).

\section{Continuous Wavelet Transform}

The Continuous Wavelet Transform (CWT) is a signal processing technique that expresses a signal as a linear combination of special functions called wavelets. These functions are scaled translations of a mother wavelet function, i.e., different widths and different $x$-axis locations of a particular function. A wavelet $w$ is required to have oscillations and is required to "die out," i.e., the function $\lim _{x \rightarrow \infty} w(x)=0$. An example of a wavelet function called the Ricker Wavelet can be seen in Figure 3A.
What results from a wavelet transform is a wavelet coefficient $W(s, \tau)$ (Equation 2), for every scale $s$ and translation (shift along the $x$-axis) $\tau$ (Leavey et al., 2003).

$$
W(s, \tau)=\frac{1}{\sqrt{s}} \int f(t) \psi^{\star}\left(\frac{t-\tau}{s}\right) d t s
$$

This essentially can be interpreted as "sliding" the wavelet of a certain width over the signal, and at each position calculating the integral of the product of the wavelet and the signal over the entire $x$-axis, producing a vector of coefficients. This process is then repeated for multiple widths of the mother wavelet. An example of the CWT applied to the SNP density of P. trichocarpa chromosome 1 can be seen in Figure 3B. Other visual examples of various mother wavelets and CWT coefficient outputs can be seen in references (Leavey et al., 2003; Mi et al., 2005; Spencer et al., 2006; Dong et al., 2008).

\section{Discrete Wavelet Transform}

The Discrete Wavelet Transform (DWT) is a sampled version of the CWT and involves sampling of the $x$ dimension of the signal and scale dimension of the wavelet (Leavey et al., 2003). This is
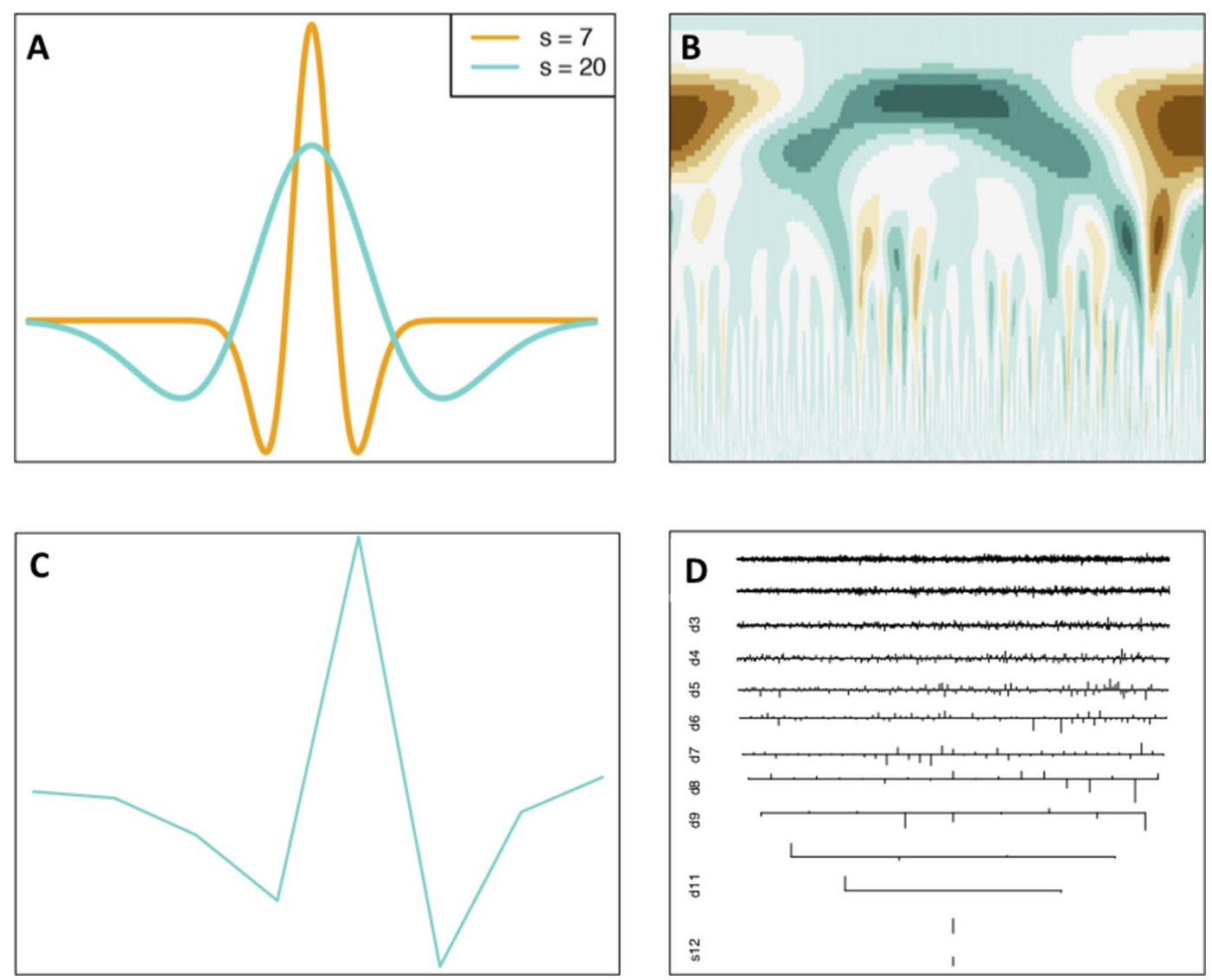

FIGURE 3 | Continuous and discrete wavelet transforms. (A) Continuous Ricker Wavelet, (B), CWT coefficient matrix heatmap, (C) discrete s8 wavelet, (D) DWT coefficients. Wavelet transform images generated using the Wavelet Methods for Time Series Analysis (WMTSA) R package (Percival and Walden, 2000). 
a dyadic sampling, which results in low-frequency, large scales being sampled sparsely and high-frequency, small scales being sampled densely (Alsberg et al., 1997). The DWT uses discrete wavelet functions (for example, see Figure 3C) and produces a series of sets of coefficients with one set of coefficients for each scale computed (Figure 3D). DWT coefficients for Palmer Drought Severity Index data across time can be seen in the tutorial by Dong et al. (2008).

\section{Wavelet-Based Analysis and Integration of Biological Data}

A useful overview of the wavelet transform and previous biological applications prior to 2003, including sequence analysis, protein structure investigation, and expression data analysis to identify periodicities, can be found in the review by Liò (2003). More recent applications of the wavelet transform in biological data analysis are discussed below.

Thurman et al. (2007) performed an investigation to detect "functional domains" of a scale larger than that of within a gene, in the human genome. The wavelet transform was used to smooth density signals of various ENCODE data over various scales. This included transcriptional data, histone acetylation, histone methylation, and DNA replication time. A hidden Markov model was then used to segment the genome into one of two states, namely, state 0 ("repressed") and state 1 ("active"), particularly signal (Thurman et al., 2007). This was performed separately for each data type and also in a combined fashion. Domains with the state 1 ("active") classification were enriched in characteristics of "active" chromatin, for example, transcriptional stop/start sites, mRNAs, and CpG islands, among others. However, domains with the state 0 ("repressed") classification were significantly enriched in signal transduction genes as determined using GO enrichment. TEs in general were evenly distributed across active and repressed domains; however, certain classes of repeats, such as L1 LINE repeats and LTR elements, were enriched in state 0 domains ("repressed" domains) (Thurman et al., 2007).

Shim and Stephens (2015) determined variants that are associated with open chromatin using DNase-seq data from 70 genotyped individuals. Chromatin accessibility vectors are transformed using the DWT prior to associating them to phenotypes. The advantage of this method is that it takes into account the read profile, without having to resort to "artificial" boundaries such as known exon boundaries or sliding windows of a set size.

Machado et al. (2011) performed wavelet analysis of sequence data by transforming DNA sequence into a vector of numbers, with each base pair mapped to a point on one of the axes of the complex plane. The wavelet transform is applied to these sequence vectors and various wavelets are tested. However, no functional interpretations of results were discussed.

Biological signals can have different relationships with each other depending on the scale at which one is looking. While two features may be correlated at certain scales, they may be anti-correlated at others. Keitt and Urban (2005) introduced wavelet-coefficient regression, in which wavelet transforms are applied to dependent and independent variables before performing regression analysis, allowing for scale-specific inference. Spencer et al. (2006) used this kind of approach, applying the DWT and linear model analysis to investigate scale-specific relationships between various genomic features including genomic signals of recombination, divergence, diversity, GC content, and gene content in 1-kb regions across human chromosome 20. The DWT was performed on each of these signals, and the correlation between the wavelet coefficients of features at each scale was calculated to identify scale-specific correlations (Spencer et al., 2006). Paape et al. (2012) applied the same approach as Spencer et al. (2006), using the wavelet transform followed by linear model analysis to identify genomic features that correlate with recombination in $M$. truncatula. The wavelet correlation results revealed a negative correlation between recombination and the distance to the centromere, which had not been found in several other organisms (Paape et al., 2012). Very recently, Fernández et al. (2018) applied the wavelet transform in an application for visualizing DNA methylation data at various scales/ resolutions.

Representation of multiple $P$. trichocarpa data layers as signals was performed by Vining et al. (2012), in which methylation density signals of various $P$. trichocarpa tissues were overlapped. In addition, methylation density signals were overlaid with gene density signals and k-mer density signals, and approximate centromere locations on a subset of the $P$. trichocarpa chromosomes were visually reported (Vining et al., 2012).

Weighill et al. (2019b) performed the first application of the wavelet transform to multi-omics data layering in Poplar. Weighill et al. (2019b) made use of the methylation data from Vining et al. (2012) and Vining et al. (2013), as well as variant data and genome annotations to construct gene density, variant density, and methylation density profiles for $P$. trichocarpa (Weighill et al., 2019b). The wavelet transform was used to characterize the variation in these signals at multiple scales, extract the relevant centromere/pericentromere scales of variation, and predict the locations of the centromere on all 19 P. trichocarpa chromosomes, making use of information from variant density and methylation density signals.

\section{CONCLUDING REMARKS AND FUTURE PROSPECTS}

In this review, we have discussed large-scale 'omics data types, multi-omics studies, as well as network-based analysis/ integration techniques and wavelet-based multi-scale analysis and comparisons, all with a particular focus on investigations performed in Populus. Table 3 summarizes examples of multiomic/data integration studies in Populus. While many such studies have been performed over the last decade, few studies involve the integration of multiple data types in a combined analysis, as opposed to a sequential analysis.

A vast collection of different data types has been generated for $P$. trichocarpa. As described in this review, the genome has been sequenced and annotated (Tuskan et al., 2006), and the assembly is currently in its fourth version of revision. 
TABLE 3 | Examples of multi-omic/data integration studies in Populus species.

\begin{tabular}{|c|c|c|}
\hline Species & Data Types/Layers & Reference \\
\hline P. trichocarpa & $\begin{array}{l}\text { Transcriptomics, metabolomics, } \\
\text { biomass/sugar release }\end{array}$ & Zhang et al. (2019) \\
\hline P. trichocarpa & $\begin{array}{l}\text { Genomic, transcriptomic, } \\
\text { proteomic, fluxomic, wood } \\
\text { chemical property phenotypes }\end{array}$ & Wang et al. (2018b) \\
\hline $\begin{array}{l}P . \text { tremula } \times P . \\
\text { tremuloides }\end{array}$ & $\begin{array}{l}\text { Transcriptome, proteome, } \\
\text { GC-MS metabolome, LC-MS } \\
\text { metabolome, pyrolysis-GC MS } \\
\text { metabolome }\end{array}$ & Obudulu et al. (2018) \\
\hline P. trichocarpa & $\begin{array}{l}\text { Transcriptomics, co-expression, } \\
\text { genotype, callus phenotype } \\
\text { (GWAS) }\end{array}$ & Tuskan et al. (2018) \\
\hline P. trichocarpa & $\begin{array}{l}\text { Metabolomics, genotype, } \\
\text { transcriptomics, GWAS, eQTL, } \\
\text { co-expression }\end{array}$ & Zhang et al. (2018) \\
\hline P. deltoides & Metabolomics, microbiome & Veach et al. (2018) \\
\hline P. trichocarpa & $\begin{array}{l}\text { Co-expression, protein-protein } \\
\text { interaction, population genotype }\end{array}$ & Tian et al. (2017) \\
\hline P. trichocarpa & $\begin{array}{l}\text { Methylation, transcript } \\
\text { expression, miRNAs }\end{array}$ & $\begin{array}{l}\text { Schönberger et al. } \\
\text { (2016) }\end{array}$ \\
\hline $\begin{array}{l}\text { P. tremuloides } \\
\text { and Laccaria }\end{array}$ & $\begin{array}{l}\text { Transcriptomics, protein-protein } \\
\text { interactions, }\end{array}$ & Larsen et al. (2016) \\
\hline P. balsamifera & Transcriptomics, metabolomics & Hamanishi et al. (2015) \\
\hline $\begin{array}{l}P . \text { trichocarpa } \\
\text { and } P \text {. } \\
\text { deltoides }\end{array}$ & Metabolomics, transcriptomics & $\begin{array}{l}\text { Tschaplinski et al. } \\
\text { (2014) }\end{array}$ \\
\hline P. trichocarpa & Genotype, phenotype (GWAS) & McKown et al. (2014) \\
\hline P. trichocarpa & $\begin{array}{l}\text { Methylome (bisulfite sequencing), } \\
\text { transcriptomics }\end{array}$ & Liang et al. (2014) \\
\hline P. trichocarpa & Genotype, phenotype (GWAS) & Evans et al. (2014) \\
\hline P. trichocarpa & $\begin{array}{l}\text { Methylome (MeDIP-seq), } \\
\text { transcriptomics }\end{array}$ & Vining et al. (2013) \\
\hline P. trichocarpa & Open chromatin, methylome & $\begin{array}{l}\text { Lafon-Placette et al. } \\
\text { (2013) }\end{array}$ \\
\hline P. trichocarpa & $\begin{array}{l}\text { Methylome (MeDIP-seq), } \\
\text { transcriptomics, transposable } \\
\text { elements }\end{array}$ & Vining et al. (2012) \\
\hline P. trichocarpa & $\begin{array}{l}\text { Genotype, repeat elements, } \\
\text { methylation, recombination }\end{array}$ & Slavov et al. (2012) \\
\hline $\begin{array}{l}\text { Populus } \\
\text { euphratica } \\
\text { and Populus } \times \\
\text { canescens }\end{array}$ & Transcriptomics, metabolomics & Janz et al. (2010) \\
\hline $\begin{array}{l}\text { P. tremula } \times P \text {. } \\
\text { tremuloides }\end{array}$ & $\begin{array}{l}\text { Transcriptomics, metabolomics, } \\
\text { proteomics }\end{array}$ & Bylesjo et al. (2008) \\
\hline $\begin{array}{l}\text { P. tremula } \times P \text {. } \\
\text { tremuloides }\end{array}$ & Transcriptomics, metabolomics & Bylesjö et al. (2007) \\
\hline $\begin{array}{l}P . \text { deltoides } \times \\
P . \text { nigra and } P \text {. } \\
\text { deltoides } \times P \text {. } \\
\text { trichocarpa }\end{array}$ & Genotypes, metabolites (mQTLs) & Morreel et al. (2006) \\
\hline
\end{tabular}

Approximately $\sim 1,300 \quad P$. trichocarpa genotypes have been propagated in four different common gardens (Tuskan et al., 2011; Slavov et al., 2012; Evans et al., 2014) and have been resequenced. This has provided a large set of $\sim 28,000,000$ single-nucleotide polymorphisms (SNPs) that have recently been publicly released (DOI 10.13139/OLCF/1411410). Many molecular phenotypes measured through untargeted metabolomics, RNA-Seq, ionomics, and pyMBMS, as well as physical properties (Porth et al., 2013) measured in this population have provided an unparalleled resource for GWASs [for example, see McKown et al. (2014)]. DNA methylation data in the form of MeDIP (Methyl-DNA immunoprecipitation)seq have been performed on 10 different $P$. trichocarpa tissues (Vining et al., 2012).

The availability of public data as well as access to highperformance computing resources provides an opportunity for the large-scale, concurrent analysis of these multiple datasets in order to profile and characterize the P. trichocarpa genome; identify complex gene-phenotype relationships, such as pleiotropy and epistasis, from genome-wide association data; as well as perform large-scale target gene identification from integrated multi-omics datasets. Multi-scale analysis could allow for the interrogation of scale-specific relationships between various genomic features and could potentially provide insights into the evolutionary history of the $P$. trichocarpa genome. Integrated analysis of various 'omics data layers will expand the system-wide knowledge of Populus species, which is necessary for the continued development of Populus as a model tree species and as a domesticated, efficient biofuel feedstock.

\section{AUTHOR CONTRIBUTIONS}

DW and DJ planned the manuscript. DW performed the research and wrote the manuscript. DJ, TT, and GT provided editorial feedback.

\section{FUNDING}

Funding was provided by the Center for Bioenergy Innovation (CBI). The Center for Bioenergy Innovation is a U.S. Department of Energy Bioenergy Research Center supported by the Office of Biological and Environmental Research in the DOE Office of Science.

This research was also supported by the Plant-Microbe Interfaces Scientific Focus Area (http://pmi.ornl.gov) in the Genomic Science Program, the Office of Biological and Environmental Research (BER) in the U.S. Department of Energy Office of Science.

An award of computer time was provided by the INCITE program. This research used resources of the Oak Ridge Leadership Computing Facility (OLCF) at the Oak Ridge National Laboratory, which is supported by the Office of Science of the U.S. Department of Energy under Contract No. DE-AC05-00OR22725.

\section{SUPPLEMENTARY MATERIAL}

The Supplementary Material for this article can be found online at: https://www.frontiersin.org/articles/10.3389/fgene.2019.00874/ full\#supplementary-material 


\section{REFERENCES}

Abel, H. J., and Duncavage, E. J. (2013). Detection of structural DNA variation from next generation sequencing data: a review of informatic approaches. Cancer Genet. 206, 432-440. doi: 10.1016/j.cancergen.2013.11.002

Abraham, P. E., Garcia, B. J., Gunter, L. E., Jawdy, S. S., Engle, N., Yang, X., et al. (2018). Quantitative proteome profile of water deficit stress responses in eastern cottonwood (Populus deltoides) leaves. PloS One 13, e0190019. doi: 10.1371/journal.pone.0190019

Akula, N., Baranova, A., Seto, D., Solka, J., Nalls, M. A., Singleton, A., et al. (2011). A network-based approach to prioritize results from genome-wide association studies. PloS One 6, e24220. doi: 10.1371/journal.pone.0024220

Akulenko, R., and Helms, V. (2013). DNA co-methylation analysis suggests novel functional associations between gene pairs in breast cancer samples. Hum. Mol. Genet. 22, 3016-3022. doi: 10.1093/hmg/ddt158

Alsberg, B. K., Woodward, A. M., and Kell, D. B. (1997). An introduction to wavelet transforms for chemometricians: a time-frequency approach. Chemom. Intell. Lab. Syst. 37, 215-239. doi: 10.1016/S0169-7439(97)00029-4

Aoki, K., Ogata, Y., and Shibata, D. (2007). Approaches for extracting practical information from gene co-expression networks in plant biology. Plant Cell Physiol. 48, 381-390. doi: 10.1093/pcp/pcm013

Arneson, D., Bhattacharya, A., Shu, L., Mäkinen, V.-P., and Yang, X. (2016). Mergeomics: a web server for identifying pathological pathways, networks, and key regulators via multidimensional data integration. BMC Genomics 17, 722. doi: 10.1186/s12864-016-3057-8

Astle, W., and Balding, D. J., (2009). Population structure and cryptic relatedness in genetic association studies. Stat. Sci. 24, 451-471. doi: 10.1214/09-STS307

Bajic M., Maher K. A., Deal R. B. (2018) "Identification of Open Chromatin Regions in Plant Genomes Using ATAC-Seq". In Plant Chromatin Dynamics. Methods in Molecular Biology. 1675. New York, NY, Humana Press. doi: 10.1007/978-1-4939-7318-7_12

Bao, H., Li, E., Mansfield, S. D., Cronk, Q. C., El-Kassaby, Y. A., and Douglas, C. J. (2013). The developing xylem transcriptome and genome-wide analysis of alternative splicing in Populus trichocarpa (black cottonwood) populations. BMC Genomics 14, 359. doi: 10.1186/1471-2164-14-359

Barabasi, A.-L., and Oltvai, Z. N. (2004). Network biology: understanding the cell's functional organization. Nat. Rev. Genet. 5, 101-113. doi: 10.1038/nrg1272

Bartlett, A., O’Malley, R. C., Huang, S.-s. C., Galli, M., Nery, J. R., Gallavotti, A., et al. (2017). Mapping genome-wide transcription-factor binding sites using DAP-seq. Nat. Protoc. 12, 1659. doi: 10.1038/nprot.2017.055

Bartlett, T. E., Olhede, S. C., and Zaikin, A. (2014). A DNA methylation network interaction measure, and detection of network oncomarkers. PloS One 9, e84573. doi: 10.1371/journal.pone.0084573

Benedito, V. A., Torres-Jerez, I., Murray, J. D., Andriankaja, A., Allen, S., Kakar, K., et al. (2008). A gene expression atlas of the model legume Medicago truncatula. Plant J. 55, 504-513. doi: 10.1111/j.1365-313X.2008.03519.x

Bennetzen, J. L., and Wang, H. (2014). The contributions of transposable elements to the structure, function, and evolution of plant genomes. Annu. Rev. Plant Biol. 65, 505-530. doi: 10.1146/annurev-arplant-050213-035811

Bock, C. (2012). Analysing and interpreting DNA methylation data. Nat. Rev. Genet. 13, 705. doi: 10.1038/nrg3273

Bryan, A. C., Zhang, J., Guo, J., Ranjan, P., Singan, V., Barry, K., et al. (2018). A variable polyglutamine repeat affects subcellular localization and regulatory activity of a Populus ANGUSTIFOLIA protein. G3: Genes, Genomes, Genet. 8, 2631-2641. doi: 10.1534/g3.118.200188

Buenrostro, J. D., Giresi, P. G., Zaba, L. C., Chang, H. Y., and Greenleaf, W. J. (2013). Transposition of native chromatin for fast and sensitive epigenomic profiling of open chromatin, DNA-binding proteins and nucleosome position. Nat. Methods 10, 1213. doi: 10.1038/nmeth.2688

Buenrostro, J. D., Wu, B., Chang, H. Y., and Greenleaf, W. J. (2015). ATAC-seq: amethod for assaying chromatin accessibility genome-wide. Curr. Protoc. Mol. Biol. 109, 21-29. doi: 10.1002/0471142727.mb2129s109

Bunyavanich, S., Schadt, E. E., Himes, B. E., Lasky-Su, J., Qiu, W., Lazarus, R., et al. (2014). Integrated genome-wide association, coexpression network, and expression single nucleotide polymorphism analysis identifies novel pathway in allergic rhinitis. BMC Med. Genomics 7, 48. doi: 10.1186/1755-8794-7-48

Bush, W. S., and Moore, J. H. (2012). Genome-wide association studies. PLoS Comput. Biol. 8, e1002822. doi: 10.1371/journal.pcbi.1002822
Bylesjö, M., Eriksson, D., Kusano, M., Moritz, T., and Trygg, J. (2007). Data integration in plant biology: the O2PLS method for combined modeling of transcript and metabolite data. Plant J. 52, 1181-1191. doi: 10.1111/j.1365-313X.2007.03293.X

Bylesjo, M., Nilsson, R., Srivastava, V., Gronlund, A., Johansson, A. I., Jansson, S., et al. (2008). Integrated analysis of transcript, protein and metabolite data to study lignin biosynthesis in hybrid aspen. J. Proteome Res. 8, 199-210. doi: $10.1021 / \mathrm{pr} 800298 \mathrm{~s}$

Calabrese, G. M., Mesner, L. D., Stains, J. P., Tommasini, S. M., Horowitz, M. C., Rosen, C. J., et al. (2017). Integrating GWAS and co-expression network data identifies bone mineral density genes SPTBN1 and MARK3 and an osteoblast functional module. Cell Syst. 4, 46-59. doi: 10.1016/j.cels.2016.10.014

Capy, P., Gasperi, G., Biémont, C., and Bazin, C. (2000). Stress and transposable elements: co-evolution or useful parasites? Heredity 85, 101. doi: 10.1046/j.1365-2540.2000.00751.x

Chao, Q., Gao, Z.-F., Zhang, D., Zhao, B.-G., Dong, F.-Q., Fu, C.-X., et al. (2019). The developmental dynamics of the Populus stem transcriptome. Plant Biotechnol. J. 17, 206-219. doi: 10.1111/pbi.12958

Chen, S., and Harmon, A. C. (2006). Advances in plant proteomics. Proteomics 6, 5504-5516. doi: 10.1002/pmic.200600143

Chhetri, H. B., Macaya-Sanz, D., Kainer, D., Biswal, A. K., Evans, L. M., Chen, J.-G., et al. (2019). Multitrait genome-wide association analysis of Populus trichocarpa identifies key polymorphisms controlling morphological and physiological traits. New Phytol. 223, 293-309. doi: 10.1111/nph.15777

Chinnusamy, V., and Zhu, J.-K. (2009). Epigenetic regulation of stress responses in plants. Curr. Opin. Plant Biol. 12, 133-139. doi: 10.1016/j.pbi.2008.12.006

Climer, S., Templeton, A. R., and Zhang, W. (2014a). Allele-specific network reveals combinatorial interaction that transcends small effects in psoriasis GWAS. PLOS Comput. Biol. 10, e1003766. doi: 10.1371/journal.pcbi.1003766

Climer, S., Templeton, A. R., and Zhang, W. (2015). Human gephyrin is encompassed within giant functional noncoding yin-yang sequences. Nat. Commun. 6, 6534. doi: 10.1038/ncomms 7534

Climer, S., Yang, W., Fuentes, L., Dávila-Román, V. G., and Gu, C. C. (2014b). A Custom Correlation Coefficient (CCC) approach for fast identification of multi-SNP association patterns in genome-wide SNPs data. Genet. Epidemiol. 38, 610-621. doi: 10.1002/gepi.21833

Cossu, R. M., Buti, M., Giordani, T., Natali, L., and Cavallini, A. (2012). A computational study of the dynamics of LTR retrotransposons in the Populus trichocarpa genome. Tree Genet. Genomes 8, 61-75. doi: 10.1007/ s11295-011-0421-3

Darabos, C., Harmon, S. H., and Moore, J. H. (2014). Using the bipartite human phenotype network to reveal pleiotropy and epistasis beyond the gene. Pac. Symp. Biocomput. (World Sci.) 19, 188-199. doi: 10.1142/9789814583220_0019

Dash, M., Yordanov, Y. S., Georgieva, T., Wei, H., and Busov, V. (2018). Gene network analysis of poplar root transcriptome in response to drought stress identifies a PtaJAZ3PtaRAP2. 6-centered hierarchical network. PloS One 13, e0208560. doi: 10.1371/journal.pone.0208560

Dimitrakopoulos, C., Hindupur, S. K., Häfliger, L., Behr, J., Montazeri, H., Hall, M. N., et al. (2018). Network-based integration of multi-omics data for prioritizing cancer genes. Bioinformatics 34, 2441-2448. doi: 10.1093/ bioinformatics/btyl48

Dong, X., Nyren, P., Patton, B., Nyren, A., Richardson, J., and Maresca, T. (2008). Wavelets for agriculture and biology: a tutorial with applications and outlook. BioScience 58, 445-453. doi: 10.1641/B580512

Druka, A., Muehlbauer, G., Druka, I., Caldo, R., Baumann, U., Rostoks, N., et al. (2006). An atlas of gene expression from seed to seed through barley development. Funct. Integr. Genomics 6, 202-211. doi: 10.1007/ s10142-006-0025-4

Durand, T. C., Sergeant, K., Renaut, J., Planchon, S., Hoffmann, L., Carpin, S., et al. (2011). Poplar under drought: comparison of leaf and cambial proteomic responses. J. Proteomics 74, 1396-1410. doi: 10.1016/j.jprot.2011.03.013

Emamjomeh, A., Robat, E. S., Zahiri, J., Solouki, M., and Khosravi, P. (2017). Gene co-expression network reconstruction: a review on computational methods for inferring functional information from plant-based expression data. Plant Biotechnol. Rep. 11, 71-86. doi: 10.1007/s11816-017-0433-z

Enright, A. J., Van Dongen, S., and Ouzounis, C. A. (2002). An efficient algorithm for large-scale detection of protein families. Nucleic Acids Res. 30, 1575-1584. doi: 10.1093/nar/30.7.1575 
Evans, L. M., Slavov, G. T., Rodgers-Melnick, E., Martin, J., Ranjan, P., Muchero, W., et al. (2014). Population genomics of Populus trichocarpa identifies signatures of selection and adaptive trait associations. Nat. Genet. 46, 1089-1096. doi: 10.1038/ng.3075

Fadista, J., Manning, A. K., Florez, J. C., and Groop, L. (2016). The (in) famous GWAS P-value threshold revisited and updated for low-frequency variants. Eur. J. Hum. Genet. 24, 1202. doi: 10.1038/ejhg.2015.269

Fagny, M., Paulson, J. N., Kuijjer, M. L., Sonawane, A. R., Chen, C.-Y., LopesRamos, C. M., et al. (2017). Exploring regulation in tissues with eQTL networks. Proc. Nat. Acad. Sci. 114, E7841-E7850. doi: 10.1073/pnas.1707375114

Farber, C. R. (2013). Systems-level analysis of genome-wide association data. G3: Genes, Genomes, Genet. 3, 119-129. doi: 10.1534/g3.112.004788

Fasoli, M., Dal Santo, S., Zenoni, S., Tornielli, G., Farina, L., Zamboni, A., Pezzotti, M. (2012). The Grapevine Expression Atlas Reveals a Deep Transcriptome Shift Driving the Entire Plant into a Maturation Program. The Plant Cell. 24(9), 3489-3505. doi: 10.1105/tpc.112.100230

Fernández, L., Pérez, M., and Orduña, J. M. (2018). Visualization of DNA methylation results through a GPU-based parallelization of the wavelet transform. J. Supercomput. 75 (3), 1496-1509. doi: 10.1007/s11227-018-2670-5

Finnegan, E., Genger, R., Peacock, W., and Dennis, E. (1998). DNA methylation in plants. Annu. Rev. Plant Biol. 49, 223-247. doi: 10.1146/annurev. arplant.49.1.223

Flint-Garcia, S. A., Thornsberry, J. M., and IV, B. (2003). Structure of linkage disequilibrium in plants. Annu. Rev. Plant Biol. 54, 357-374 doi: 10.1146/ annurev.arplant.54.031902.134907

Frommer, M., McDonald, L. E., Millar, D. S., Collis, C. M., Watt, F., Grigg, G. W., et al. (1992). A genomic sequencing protocol that yields a positive display of 5-methylcytosine residues in individual DNA strands. Proc. Nat. Acad. Sci. 89, 1827-1831. doi: 10.1073/pnas.89.5.1827

Fukushima, A., and Kusano, M. (2014). A network perspective on nitrogen metabolism from model to crop plants using integrated 'omics' approaches. J. Exp. Bot. 65, 5619-5630. doi: 10.1093/jxb/eru322

Gaut, B. S., Wright, S. I., Rizzon, C., Dvorak, J., and Anderson, L. K. (2007). Recombination: an underappreciated factor in the evolution of plant genomes. Nat. Rev. Genet. 8, 77. doi: 10.1038/nrg1970

Gene Ontology Consortium. (2004). The Gene Ontology (GO) database and informatics resource. Nucleic Acids Res. 32, D258-D261. doi: 10.1093/nar/ gkh036

Geraldes, A., Difazio, S., Slavov, G., Ranjan, P., Muchero, W., and Hannemann, J. (2013). A 34K SNP genotyping array for Populus trichocarpa: design, application to the study of natural populations and transferability to other Populus species. Mol. Ecol. Resour. 13, 306-323. doi: 10.1111/1755-0998.12056

Gligorijević, V., and Pržulj, N. (2015). Methods for biological data integration: perspectives and challenges. J. Royal Soc. Interface 12, 20150571. doi: 10.1098/ rsif.2015.0571

Goh, K.-I., Cusick, M. E., Valle, D., Childs, B., Vidal, M., and Barabási, A.-L. (2007). The human disease network. Proc. Nat. Acad. Sci. 104, 8685-8690. doi: $10.1073 /$ pnas. 0701361104

Golumbic, M. (2004).“Annals of discrete mathematics" in Algorithmic graph theory and perfect graphs. 2nd ed. Amsterdam; Boston: Elsevier. doi: 10.1016/ S0167-5060(04)80059-1

Goodstein, D. M., Shu, S., Howson, R., Neupane, R., Hayes, R. D., Fazo, J., et al. (2012). Phytozome: a comparative platform for green plant genomics. Nucleic Acids Res. 40, D1178-D1186. doi: 10.1093/nar/gkr944

Grönlund, A., Bhalerao, R. P., and Karlsson, J. (2009). Modular gene expression in Poplar: a multilayer network approach. New Phytol. 181, 315-322. doi: 10.1111/j.1469-8137.2008.02668.x

Gutiérrez, R. A., Lejay, L. V., Dean, A., Chiaromonte, F., Shasha, D. E., and Coruzzi, G. M. (2007). Qualitative network models and genome-wide expression data define carbon/nitrogen-responsive molecular machines in Arabidopsis. Genome Biol. 8, R7. doi: 10.1186/gb-2007-8-1-r7

Ha, M. J., Baladandayuthapani, V., and Do, K.-A. (2015). DINGO: differential network analysis in genomics. Bioinformatics 31, 3413-3420. doi: 10.1093/ bioinformatics/btv406

Hamanishi, E. T., Barchet, G. L., Dauwe, R., Mansfield, S. D., and Campbell, M. M. (2015). Poplar trees reconfigure the transcriptome and metabolome in response to drought in a genotype-and time-of-day-dependent manner. BMC Genomics 16, 329. doi: $10.1186 / \mathrm{s} 12864-015-1535-\mathrm{z}$
Hassani-Pak K. (2017) KnetMiner - An integrated data platform for gene mining and biological knowledge discovery. Bielefeld: Universität Bielefeld.

Hassani-Pak, K., Castellote, M., Esch, M., Hindle, M., Lysenko, A., Taubert, J., et al. (2016). Developing integrated crop knowledge networks to advance candidate gene discovery. Appl. Transl. Genomics 11, 18-26. doi: 10.1016/j. atg.2016.10.003

Hassani-Pak, K., and Rawlings, C. (2017). Knowledge discovery in biological databases for revealing candidate genes linked to complex phenotypes. J. Integr. Bioinf. 14 (1), 803-809. doi: 10.1515/jib-2016-0002

Horvath, S., and Dong, J. (2008). Geometric interpretation of gene coexpression network analysis. PLoS Comput. Biol. 4, e1000117. doi: 10.1371/journal. pcbi. 1000117

Hu, H., Scheben, A., and Edwards, D. (2018). Advances in integrating genomics and bioinformatics in the plant breeding pipeline. Agriculture 8, 75. doi: 10.3390/agriculture 8060075

Ingvarsson, P. K., Hvidsten, T. R., and Street, N. R. (2016). Towards integration of population and comparative genomics in forest trees. New Phytol. 212, 338344. doi: $10.1111 / \mathrm{nph} .14153$

Jacinto, F. V., Ballestar,E., and Esteller, M. (2008). Methyl-DNA immunoprecipitation (MeDIP): hunting down the DNA methylome. Biotechniques 44, 35-43. doi: $10.2144 / 000112708$

Jansson, S., and Douglas, C. J. (2007). Populus: a model system for plant biology. Annu. Rev. Plant Biol. 58, 435-458. doi: 10.1146/annurev. arplant.58.032806.103956

Janz, D., Behnke, K., Schnitzler, J.-P., Kanawati, B., Schmitt-Kopplin, P., and Polle, A. (2010). Pathway analysis of the transcriptome and metabolome of salt sensitive and tolerant poplar species reveals evolutionary adaption of stress tolerance mechanisms. BMC Plant Biol. 10, 150. doi: 10.1186/1471-2229-10-150

Johnson, R. C., Nelson, G. W., Troyer, J. L., Lautenberger, J. A., Kessing, B. D., Winkler, C. A., et al. (2010). Accounting for multiple comparisons in a genome-wide association study (GWAS). BMC Genomics 11, 724. doi: 10.1186/1471-2164-11-724

Joubert, W., Nance, J., Climer, S., Weighill, D., and Jacobson, D. (2019). Parallel accelerated custom correlation coefficient calculations for genomics applications. Parallel Comput. 84, 15-23. doi: 10.1016/j.parco.2019.02.003

Joubert, W., Weighill, D., Kainer, D., Climer, S., Justice, A., Fagnan, K. et al., (2018). Attacking the opioid epidemic: determining the epistatic and pleiotropic genetic architectures for chronic pain and opioid addiction. SC '18 Proceedings of the International Conference for High Performance Computing, Networking, Storage, and Analysis; 2018 November 11-16; NJ, USA: IEEE Press Piscataway, 57. doi: $10.1109 /$ SC.2018.00060

Kaling, M., Kanawati, B., Ghirardo, A., Albert, A., Winkler, J. B., Heller, W. et al. (2015). UV-B mediated metabolic rearrangements in poplar revealed by non-targeted metabolomics. Plant. Cell Environ. 38, 892-904. doi: 10.1111/ pce. 12348

Keitt, T. H., and Urban, D. L. (2005). Scale-specific inference using wavelets. Ecology 86, 2497-2504. doi: 10.1890/04-1016

Kejnovsky, E., Hawkins, J. S., and Feschotte, C. (2012). "Plant transposable elements: biology and evolution," in Plant Genome Diversity. 1, 17-34. Vienna: Springer. doi: 10.1007/978-3-7091-1130-7_2

Klein, S. J., and O'Neill, R. J. (2018). Transposable elements: genome innovation, chromosome diversity, and centromere conflict. Chromosome Res., 26(1-2), 5-23. doi: 10.1007/s10577-017-9569-5

Kogelman, L. J., and Kadarmideen, H. N. (2014). Weighted Interaction SNP Hub (WISH) network method for building genetic networks for complex diseases and traits using whole genome genotype data. BMC Syst. Biol. 8, S5. doi: 10.1186/1752-0509-8-S2-S5

Korte, A., and Farlow, A. (2013). The advantages and limitations of trait analysis with GWAS: a review. Plant Methods 9, 29. doi: 10.1186/1746-4811-9-29

Krishnan, A., Taroni, J. N., and Greene, C. S. (2016). Integrative networks illuminate biological factors underlying gene-disease associations. Curr. Genet. Med. Rep. 4, 155-162. doi: 10.1007/s40142-016-0102-5

Krueger, F., Kreck, B., Franke, A., and Andrews, S. R. (2012). DNA methylome analysis using short bisulfite sequencing data. Nat. Methods 9, 145. doi: $10.1038 /$ nmeth. 1828

Lafon-Placette, C., Faivre-Rampant, P., Delaunay, A., Street, N., Brignolas, F., and Maury, S. (2013). Methylome of DNase I sensitive chromatin in Populus trichocarpa shoot apical meristematic cells: a simplified approach revealing 
characteristics of gene-body DNA methylation in open chromatin state. New Phytol. 197, 416-430. doi: 10.1111/nph.12026

LaFramboise, T. (2009). Single nucleotide polymorphism arrays: a decade of biological, computational and technological advances. Nucleic Acids Res. 37, 4181-4193. doi: 10.1093/nar/gkp552

Laird, P. W. (2010). Principles and challenges of genome-wide DNA methylation analysis. Nat. Rev. Genet. 11, 191. doi: 10.1038/nrg2732

Lämke, J., and Bäurle, I. (2017). Epigenetic and chromatin-based mechanisms in environmental stress adaptation and stress memory in plants. Genome Biol. 18, 124. doi: 10.1186/s13059-017-1263-6

Langfelder, P., and Horvath, S. (2008). WGCNA: an R package for weighted correlation network analysis. BMC Bioinf. 9, 559. doi: 10.1186/1471-2105-9-559

Larsen, P. E., Sreedasyam, A., Trivedi, G., Desai, S., Dai, Y., Cseke, L. J., et al. (2016). Multi-omics approach identifies molecular mechanisms of plant-fungus mycorrhizal interaction. Front. Plant Sci. 6, 1061. doi: 10.3389/fpls.2015.01061

Law, J. A., and Jacobsen, S. E. (2010). Establishing, maintaining and modifying DNA methylation patterns in plants and animals. Nat. Rev. Genet. 11, 204. doi: 10.1038/nrg2719

Leavey, C., James, M., Summerscales, J., and Sutton, R. (2003). An introduction to wavelet transforms: a tutorial approach. Insight-Non-Destr. Test. Condition Monit. 45, 344-353. doi: 10.1784/insi.45.5.344.52875

Lee, T., Kim, H., and Lee, I. (2015). Network-assisted crop systems genetics: network inference and integrative analysis. Curr. Opin. Plant Biol. 24, 61-70. doi: 10.1016/j.pbi.2015.02.001

Leiserson, M. D., Eldridge, J. V., Ramachandran, S., and Raphael, B. J. (2013). Network analysis of GWAS data. Curr. Opin. Genet. Dev. 23, 602-610. doi: 10.1016/j.gde.2013.09.003

Levine M.E., Langfelder P., Horvath S. (2017) A Weighted SNP Correlation Network Method for Estimating Polygenic Risk Scores. In: Tatarinova T., Nikolsky Y. (eds) Biological Networks and Pathway Analysis. Methods in Molecular Biology, vol 1613. New York, NY, Humana Press.

Li, Y., Pearl, S. A., and Jackson, S. A. (2015). Gene networks in plant biology: approaches in reconstruction and analysis. Trends Plant Sci. 20, 664-675. doi: 10.1016/j.tplants.2015.06.013

Liang, D., Zhang, Z., Wu, H., Huang, C., Shuai, P., Ye, C.-Y., et al. (2014). Singlebase-resolution methylomes of Populus trichocarpa reveal the association between DNA methylation and drought stress. BMC Genet. 15, S9. doi: 10.1186/1471-2156-15-S1-S9

Liò, P. (2003). Wavelets in bioinformatics and computational biology: State of art and perspectives. Bioinformatics 19, 2-9. doi: 10.1093/bioinformatics/19.1.2

Liu, C., Lu, F., Cui, X., and Cao, X. (2010). Histone methylation in higher plants. Annu. Rev. Plant Biol. 61, 395-420. doi: 10.1146/annurev.arplant.043008.091939

Liu, J., Hai, G., Wang, C., Cao, S., Xu, W., Jia, Z., et al. (2015). Comparative proteomic analysis of Populus trichocarpa early stem from primary to secondary growth. J. Proteomics 126, 94-108. doi: 10.1016/j.jprot.2015.05.032

Liu, J., Ye, M., Zhu, S., Jiang, L., Sang, M., Gan, J., et al. (2018). Two-stage identification of SNP effects on dynamic poplar growth. Plant J. 93, 286-296. doi: 10.1111/tpj.13777

Liu, Q., Ding, C., Chu, Y., Chen, J., Zhang, W., Zhang, B., et al. (2016a). PoplarGene: poplar gene network and resource for mining functional information for genes from woody plants. Sci. Rep. 6, 31356. doi: 10.1038/srep31356

Liu, Y., Beyer, A., and Aebersold, R. (2016b). On the dependency of cellular protein levels on mRNA abundance. Cell 165, 535-550. doi: 10.1016/j.cell.2016.03.014

Lu, Z., Hofmeister, B. T., Vollmers, C., DuBois, R. M., and Schmitz, R. J. (2016). Combining ATAC-seq with nuclei sorting for discovery of cis-regulatory regions in plant genomes. Nucleic Acids Res. 45, e41-e41. doi: 10.1093/nar/ gkw1179

Luo, J., Xia, W., Cao, P., Xiao, Z., Zhang, Y., Liu, M., et al. (2019). Integrated transcriptome analysis reveals plant hormones jasmonic acid and salicylic acid coordinate growth and defense responses upon fungal infection in poplar. Biomolecules 9, 12. doi: 10.3390/biom9010012

Lusser, A., Kölle, D., and Loidl, P. (2001). Histone acetylation: lessons from the plant kingdom. Trends Plant Sci. 6, 59-65. doi: 10.1016/S1360-1385(00)01839-2

Machado, J. T., Costa, A. C., and Quelhas, M. D. (2011). Wavelet analysis of human DNA. Genomics 98, 155-163. doi: 10.1016/j.ygeno.2011.05.010

Maher, K. A., Bajic, M., Kajala, K., Reynoso, M., Pauluzzi, G., West, D. A., et al. (2018). Profiling of accessible chromatin regions across multiple plant species and cell types reveals common gene regulatory principles and new control modules. Plant Cell 30, 15-36. doi: 10.1105/tpc.17.00581

Mascagni, F., Usai, G., Natali, L., Cavallini, A., and Giordani, T. (2018). A comparison of methods for LTR-retrotransposon insertion time profiling in the Populus trichocarpa genome. Caryologia 71, 85-92. doi: 10.1080/ 00087114.2018.1429749

McCormick, R. F., Truong, S. K., Sreedasyam, A., Jenkins, J., Shu, S., Sims, D., et al. (2018). The Sorghum bicolor reference genome: improved assembly, gene annotations, a transcriptome atlas, and signatures of genome organization. Plant J. 93, 338-354. doi: 10.1111/tpj.13781

McKenna, A., Hanna, M., Banks, E., Sivachenko, A., Cibulskis, K., Kernytsky, A., et al. (2010). The Genome Analysis Toolkit: a MapReduce framework for analyzing next-generation DNA sequencing data. Genome Res. 20 (9), 1297- 1303. doi: 10.1101/gr.107524.110

McKown, A. D., Klápště, J., Guy, R. D., Geraldes, A., Porth, I., Hannemann, J., et al. (2014). Genome-wide association implicates numerous genes underlying ecological trait variation in natural populations of Populus trichocarpa. New Phytol. 203, 535-553. doi: 10.1111/nph.12815

Mering, C.v., Huynen, M., Jaeggi, D., Schmidt, S., Bork, P., and Snel, B. (2003). STRING: a database of predicted functional associations between proteins. Nucleic Acids Res. 31, 258-261. doi: 10.1093/nar/gkg034

Mi, X., Ren, H., Ouyang, Z., Wei, W., and Ma, K. (2005). The use of the Mexican Hat and the Morlet wavelets for detection of ecological patterns. Plant Ecol. 179, 1-19. doi: 10.1007/s11258-004-5089-4

Mizrachi, E., Verbeke, L., Christie, N., Fierro, A. C., Mansfield, S. D., Davis, M. F., et al. (2017). Network-based integration of systems genetics data reveals pathways associated with lignocellulosic biomass accumulation and processing. Proc. Nat. Acad. Sci. 114, 1195-1200. doi: 10.1073/pnas.1620119114

Morreel, K., Goeminne, G., Storme, V., Sterck, L., Ralph, J., Coppieters, W., et al. (2006). Genetical metabolomics of flavonoid biosynthesis in Populus: a case study. Plant J. 47, 224-237. doi: 10.1111/j.1365-313X.2006.02786.x

Movahedi, S., VanBel, M., Heyndrickx, K. S., and Vandepoele, K. (2012). Comparative co-expression analysis in plant biology. Plant Cell Environ. 35, 1787-1798. doi: 10.1111/j.1365-3040.2012.02517.x

Natali, L., Cossu, R. M., Mascagni, F., Giordani, T., and Cavallini, A. (2015). A survey of Gypsy and Copia LTR-retrotransposon superfamilies and lineages and their distinct dynamics in the Populus trichocarpa (L.) genome. Tree Genet. Genomes 11, 107. doi: 10.1007/s11295-015-0937-z

Netotea, S., Sundell, D., Street, N. R., and Hvidsten, T. R. (2014). ComPlEx: conservation and divergence of co-expression networks in A. thaliana, Populus and O. sativa. BMC Genomics 15, 106. doi: 10.1186/1471-2164-15-106

Nielsen, R., Paul, J. S., Albrechtsen, A., and Song, Y. S. (2011). Genotype and SNP calling from next-generation sequencing data. Nat. Rev. Genet. 12, 443. doi: $10.1038 / \mathrm{nrg} 2986$

Noble, W. S. (2009). How does multiple testing correction work? Nat. Biotechnol. 27, 1135. doi: 10.1038/nbt1209-1135

Obudulu, O., Bygdell, J., Sundberg, B., Moritz, T., Hvidsten, T. R., Trygg, J., et al. (2016). Quantitative proteomics reveals protein profiles underlying major transitions in aspen wood development. BMC Genomics 17, 119. doi: 10.1186/ s12864-016-2458-z

Obudulu, O., Mähler, N., Skotare, T., Bygdell, J., Abreu, I. N., Ahnlund, M., et al. (2018). A multi-omics approach reveals function of secretory carrier-associated membrane proteins in wood formation of Populus trees. BMC Genomics 19, 11. doi: $10.1186 / \mathrm{s} 12864-017-4411-1$

Ogata, Y., Suzuki, H., and Shibata, D. (2009). A database for poplar gene co-expression analysis for systematic understanding of biological processes, including stress responses. J. Wood Sci. 55, 395. doi: 10.1007/s10086-009-1058-9

O’Malley, R. C., Huang, S.-s. C., Song, L., Lewsey, M. G., Bartlett, A., Nery, J. R., et al. (2016). Cistrome and epicistrome features shape the regulatory DNA landscape. Cell 165, 1280-1292. doi: 10.1016/j.cell.2016.04.038

Paape, T., Zhou, P., Branca, A., Briskine, R., Young, N., and Tiffin, P. (2012). Fine-scale population recombination rates, hotspots, and correlates of recombination in the Medicago truncatula genome. Genome Biol. Evol. 4, 726737. doi: $10.1093 /$ gbe/evs046

Patel, R. V., Nahal, H. K., Breit, R., and Provart, N. J. (2012). BAR expressolog identification: expression profile similarity ranking of homologous genes in plant species. Plant J. 71, 1038-1050. doi: 10.1111/j.1365-313X.2012.05055.x 
Patti, G. J., Yanes, O., and Siuzdak, G. (2012). Innovation: Metabolomics: The apogee of the omics trilogy. Nat. Rev. Mol. Cell Biol. 13, 263. doi: 10.1038/ nrm3314

Percival, D., and Walden, A. (2000). Wavelet methods for time series analysis. West Nyack: Cambridge University Press.

Porth, I., Klápště, J., Skyba, O., Lai, B. S., Geraldes, A., Muchero, W., et al. (2013). Populus trichocarpa cell wall chemistry and ultrastructure trait variation, genetic control and genetic correlations. New Phytol. 197, 777-790. doi: 10.1111/nph.12014

Quesada, T., Li, Z., Dervinis, C., Li, Y., Bocock, P. N., Tuskan, G. A., et al. (2008). Comparative analysis of the transcriptomes of Populus trichocarpa and Arabidopsis thaliana suggests extensive evolution of gene expression regulation in angiosperms. New Phytol. 180, 408-420. doi: 10.1111/j. 1469-8137.2008.02586.x

Ragauskas, A. J., Williams, C. K., Davison, B. H., Britovsek, G., Cairney, J., Eckert, C. A., et al. (2006). The path forward for biofuels and biomaterials. Science 311, 484489. doi: $10.1126 /$ science. 1114736

Ritchie, M. D., Holzinger, E. R., Li, R., Pendergrass, S. A., and Kim, D. (2015). Methods of integrating data to uncover genotype-phenotype interactions. Nat. Rev. Genet. 16, 85. doi: 10.1038/nrg3868

Sannigrahi, P., Ragauskas, A. J., and Tuskan, G. A. (2010). Poplar as a feedstock for biofuels: a review of compositional characteristics. Biofuels Bioprod. Biorefin. 4, 209-226. doi: 10.1002/bbb.206

Schaefer, R. J., Michno, J.-M., and Myers, C. L. (2017). Unraveling gene function in agricultural species using gene co-expression networks. Biochim. Biophys. Acta (BBA)-Gene Regul. Mech. 1860, 53-63. doi: 10.1016/j.bbagrm.2016.07.016

Schmid, M., Davison, T. S., Henz, S. R., Pape, U. J., Demar, M., Vingron, M., et al. (2005). A gene expression map of Arabidopsis thaliana development. Nat. Genet. 37, 501. doi: 10.1038/ng1543

Schönberger, B., Chen, X., Mager, S., and Ludewig, U. (2016). Site-dependent differences in DNA methylation and their impact on plant establishment and phosphorus nutrition in Populus trichocarpa. PloS One 11, e0168623. doi: 10.1371/journal.pone.0168623

Serin, E. A., Nijveen, H., Hilhorst, H. W., and Ligterink, W. (2016). Learning from co-expression networks: possibilities and challenges. Front. Plant Sci. 7, 444. doi: 10.3389/fpls.2016.00444

Severin, A. J., Woody, J. L., Bolon, Y.-T., Joseph, B., Diers, B. W., Farmer, A. D., et al. (2010). RNA-Seq Atlas of Glycine max: a guide to the soybean transcriptome. BMC Plant Biol. 10, 160. doi: 10.1186/1471-2229-10-160

Shannon, P., Markiel, A., Ozier, O., Baliga, N. S., Wang, J. T., Ramage, D., et al. (2003). Cytoscape: a software environment for integrated models of biomolecular interaction networks. Genome Res. 13, 2498-2504. doi: 10.1101/ gr.1239303

Shi, R., Sun, Y.-H., Li, Q., Heber, S., Sederoff, R., and Chiang, V. L. (2009). Towards a systems approach for lignin biosynthesis in Populus trichocarpa: transcript abundance and specificity of the monolignol biosynthetic genes. Plant Cell Physiol. 51, 144-163. doi: 10.1093/pcp/pcp175

Shim, H., and Stephens, M. (2015). Wavelet-based genetic association analysis of functional phenotypes arising from high-throughput sequencing assays. Ann. Appl. Stat. 9, 655. doi: 10.1214/14-AOAS776

Shuai, P., Liang, D., Tang, S., Zhang, Z., Ye, C.-Y., Su, Y., et al. (2014). Genomewide identification and functional prediction of novel and drought-responsive lincRNAs in Populus trichocarpa. J. Exp. Bot. 65, 4975-4983. doi: 10.1093/jxb/ eru256

Shuai, P., Liang, D., Zhang, Z., Yin, W., and Xia, X. (2013). Identification of droughtresponsive and novel Populus trichocarpa microRNAs by high-throughput sequencing and their targets using degradome analysis. BMC Genomics 14, 233. doi: 10.1186/1471-2164-14-233

Skinner, M. E., Uzilov, A. V., Stein, L. D., Mungall, C. J., and Holmes, I. H. (2009). JBrowse: a next-generation genome browser. Genome Res. 19, 1630-1638. doi: 10.1101/gr.094607.109

Slavov, G. T., DiFazio, S. P., Martin, J., Schackwitz, W., Muchero, W., RodgersMelnick, E., et al. (2012). Genome resequencing reveals multiscale geographic structure and extensive linkage disequilibrium in the forest tree Populus trichocarpa. New Phytol. 196, 713-725. doi: 10.1111/j.1469-8137.2012.04258.x

Slotkin, R. K., and Martienssen, R. (2007). Transposable elements and the epigenetic regulation of the genome. Nat. Rev. Genet. 8, 272. doi: 10.1038/ $\operatorname{nrg} 2072$
Solovieff, N., Cotsapas, C., Lee, P. H., Purcell, S. M., and Smoller, J. W. (2013). Pleiotropy in complex traits: challenges and strategies. Nat. Rev. Genet. 14, 483-495. doi: 10.1038/nrg3461

Spencer, C. C., Deloukas, P., Hunt, S., Mullikin, J., Myers, S., Silverman, B., et al. (2006). The influence of recombination on human genetic diversity. PLoS Genet. 2, e148. doi: 10.1371/journal.pgen.0020148

Suzuki, M. M., and Bird, A. (2008). DNA methylation landscapes: provocative insights from epigenomics. Nat. Rev. Genet. 9, 465. doi: 10.1038/nrg2341

Szklarczyk, D., Franceschini, A., Kuhn, M., Simonovic, M., Roth, A., Minguez, P., et al. (2010). The STRING database in 2011: functional interaction networks of proteins, globally integrated and scored. Nucleic Acids Res. 39, D561-D568. doi: 10.1093/nar/gkq973

Szklarczyk, D., Morris, J. H., Cook, H., Kuhn, M., Wyder, S., Simonovic, M., et al. (2017). The STRING database in 2017: Quality-controlled protein-protein association networks, made broadly accessible. Nucleic Acids Res. 45(D1), D362-D368. doi: 10.1093/nar/gkw937

Tang, S., Dong, Y., Liang, D., Zhang, Z., Ye, C.-Y., Shuai, P., et al. (2015). Analysis of the drought stress-responsive transcriptome of black cottonwood (Populus trichocarpa) using deep RNA sequencing. Plant Mol. Biol. Rep. 33, 424-438. doi: 10.1007/s11105-014-0759-4

Taylor, G. (2002). Populus Arabidopsis for Forestry. Do We Need a Model Tree?, Ann. Bot. 90 (6), 681-689. doi: 10.1093/aob/mcf255

Thimm, O., Bläsing, O., Gibon, Y., Nagel, A., Meyer, S., Krüger, P., et al. (2004). MAPMAN: a user-driven tool to display genomics data sets onto diagrams of metabolic pathways and other biological processes. Plant J. 37, 914-939. doi: 10.1111/j.1365-313X.2004.02016.x

Thurman, R. E., Day, N., Noble, W. S., and Stamatoyannopoulos, J. A. (2007). Identification of higher-order functional domains in the human ENCODE regions. Genome Res. 17, 917-927. doi: 10.1101/gr.6081407

Tian, F., Chang, E., Li, Y., Sun, P., Hu, J., and Zhang, J. (2017). Expression and integrated network analyses revealed functional divergence of NHXtype $\mathrm{Na}+\mathrm{H}+$ exchanger genes in poplar. Sci. Rep. 7, 2607. doi: 10.1038/ s41598-017-02894-8

Tschaplinski, T. J., Abraham, P. E., Jawdy, S. S., Gunter, L. E., Martin, M. Z., Engle, N. L., et al. (2019). The nature of the progression of drought stress drives differential metabolomic responses in Populus deltoides. Ann. Bot. mcz002. doi: 10.1093/aob/mcz002

Tschaplinski, T. J., Plett, J. M., Engle, N. L., Deveau, A., Cushman, K. C., Martin, M. Z., et al. (2014). Populus trichocarpa and Populus deltoides exhibit different metabolomic responses to colonization by the symbiotic fungus Laccaria bicolor. Mol. Plant-Microbe Interact. 27, 546-556. doi: 10.1094/MPMI-09-13-0286-R

Tuskan, G. (2007). Bioenergy, genomics, and accelerated domestication: a US example. FAO, Papers and Presentations from The Role of Agricultural Biotechnologies for Production of Bioenergy in Developing Countries, http:// www.fao.org/biotech/seminaroct2007.htm.

Tuskan, G., Slavov, G., DiFazio, S., Muchero, W., Pryia, R., Schackwitz, W. et al. (2011). "Populus resequencing: Towards genome-wide association studies. BMC Proceedings, 5 (Suppl 7), (BioMed Central Ltd), I21. doi: 10.1186/1753-6561-5-S7-I21

Tuskan, G. A., DiFazio, S., Faivre-Rampant, P., Gaudet, M., Harfouche, A., Jorge, V., et al. (2012). The obscure events contributing to the evolution of an incipient sex chromosome in Populus: a retrospective working hypothesis. Tree Genet. Genomes 8, 559-571. doi: 10.1007/s11295-012-0495-6

Tuskan, G. A., Difazio, S., Jansson, S., Bohlmann, J., Grigoriev, I., Hellsten, U., et al. (2006). The genome of black cottonwood, Populus trichocarpa (Torr. \& Gray). Science 313, 1596-1604. doi: 10.1126/science.1128691

Tuskan, G. A., Mewalal, R., Gunter, L. E., Palla, K. J., Carter, K., Jacobson, D. A., et al. (2018). Defining the genetic components of callus formation: a GWAS approach. PloS One 13, e0202519. doi: 10.1371/journal.pone.0202519

Usai, G., Mascagni, F., Natali, L., Giordani, T., and Cavallini, A. (2017). Comparative genome-wide analysis of repetitive DNA in the genus Populus L. Tree Genetics \& Genomes 13, 96. doi: 10.1007/s11295-017-1181-5

Valledor, L., Carbó, M., Lamelas, L., Escandón, M., Colina, F. J., Cañal, M. J., et al. (2018). When the Tree Let Us See the Forest: Systems Biology and Natural Variation Studies in Forest Species. In: Progress in Botany. Springer, Berlin: Heidelberg

van Eijk, K. R., de Jong, S., Boks, M. P., Langeveld, T., Colas, F., Veldink, J. H., et al. (2012). Genetic analysis of DNA methylation and gene expression 
levels in whole blood of healthy human subjects. BMC Genomics 13, 636. doi: 10.1186/1471-2164-13-636

Veach, A. M., Yip, D., Engle, N. L., Yang, Z. K., Bible, A., Morrell-Falvey, J., et al. (2018). Modification of plant cell wall chemistry impacts metabolome and microbiome composition in Populus PdKOR1 RNAi plants. Plant Soil 429(1- 2), 349-361. doi: 10.1007/s11104-018-3692-8

Verdier, J., Torres-Jerez, I., Wang, M., Andriankaja, A., Allen, S. N., He, J., et al. (2013). Establishment of the Lotus japonicus Gene Expression Atlas (LjGEA) and its use to explore legume seed maturation. Plant J. 74, 351-362. doi: 10.1111/tpj.12119

Vining, K., Pomraning, K. R., Wilhelm, L. J., Ma, C., Pellegrini, M., Di, Y., et al. (2013). Methylome reorganization during in vitro dedifferentiation and regeneration of Populus trichocarpa. BMC Plant Biol. 13, 92. doi: 10.1186/1471-2229-13-92

Vining, K. J., Pomraning, K. R., Wilhelm, L. J., Priest, H. D., Pellegrini, M., Mockler, T. C., et al. (2012). Dynamic DNA cytosine methylation in the Populus trichocarpa genome: tissue-level variation and relationship to gene expression. BMC Genomics 13, 1. doi: 10.1186/1471-2164-13-27

Visscher, P. M., Brown, M. A., McCarthy, M. I., and Yang, J. (2012). Five years of GWAS discovery. Am. J. Hum. Genet. 90, 7-24. doi: 10.1016/j.ajhg.2011.11.029

Visscher, P. M., Wray, N. R., Zhang, Q., Sklar, P., McCarthy, M. I., Brown, M. A., et al. (2017). 10 years of GWAS discovery: biology, function, and translation. Am. J. Hum. Genet. 101, 5-22. doi: 10.1016/j.ajhg.2017.06.005

Walley, J. W., Sartor, R. C., Shen, Z., Schmitz, R. J., Wu, K. J., Urich, M. A., et al. (2016). Integration of omic networks in a developmental atlas of maize. Science 353, 814-818. doi: 10.1126/science.aag 1125

Wang, H., Yan, H., Liu, H., Liu, R., Chen, J., and Xiang, Y. (2018a). GFDP: the gene family database in poplar. Database 2018. doi: 10.1093/database/ bayl07

Wang, J. P., Matthews, M. L., Williams, C. M., Shi, R., Yang, C., Tunlaya-Anukit, S., et al. (2018b). Improving wood properties for wood utilization through multiomics integration in lignin biosynthesis. Nat. Commun. 9, 1579. doi: 10.1038/ s41467-018-03863-Z

Wang, L., Xie, W., Chen, Y., Tang, W., Yang, J., Ye, R., et al. (2010). A dynamic gene expression atlas covering the entire life cycle of rice. Plant J. 61, 752-766. doi: 10.1111/j.1365-313X.2009.04100.x

Weckwerth, W. (2011). Green systems biology-From single genomes, proteomes and metabolomes to ecosystems research and biotechnology. J. Proteomics 75, 284-305. doi: 10.1016/j.jprot.2011.07.010

Weighill, D., Jones, P., Bleker, C., Ranjan, P., Shah, M., Zhao, N., et al. (2019a). Multi-phenotype association decomposition: unraveling complex genephenotype relationships. Front. Genet. 10, 417. doi: 10.3389/fgene.2019.00417

Weighill, D., Jones, P., Shah, M., Ranjan, P., Muchero, W., Schmutz, J., et al. (2018). Pleiotropic and epistatic network-based discovery: integrated networks for target gene discovery. Front. Energy Res. 6, 30. doi: 10.3389/fenrg.2018.00030

Weighill, D., Macaya-Sanz, D., DiFazio, S. P., Joubert, W., Shah, M., Schmutz, J., et al. (2019b). Wavelet-based genomic signal processing for centromere identification and hypothesis generation. Front. Genet. 10, 487 doi: 10.3389/ fgene.2019.00487

Weighill, D. A., and Jacobson, D. (2017). Network metamodeling: Effect of correlation metric choice on phylogenomic and transcriptomic network topology. Cham: Springer International Publishing, 143-183. doi: 10.1007/10_2016_46

Wicker, T., Sabot, F., Hua-Van, A., Bennetzen, J. L., Capy, P., Chalhoub, B., et al. (2007). A unified classification system for eukaryotic transposable elements. Nat. Rev. Genet. 8, 973. doi: 10.1038/nrg2165
Wullschleger, S. D., Weston, D., DiFazio, S. P., and Tuskan, G. A. (2012). Revisiting the sequencing of the first tree genome: Populus trichocarpa. Tree Physiol. 33, 357-364. doi: 10.1093/treephys/tps081

Xue, L.-J., Frost, C. J., Tsai, C.-J., and Harding, S. A. (2016). Drought response transcriptomes are altered in poplar with reduced tonoplast sucrose transporter expression. Sci. Rep. 6, 33655. doi: 10.1038/srep33655

Yi, F., Jia, Z., Xiao, Y., Ma, W., and Wang, J. (2018). SPTEdb: A database for transposable elements in salicaceous plants. Database 2018. doi: 10.1093/ database/bay024

Yin, T., DiFazio, S. P., Gunter, L. E., Zhang, X., Sewell, M. M., Woolbright, S. A., et al. (2008). Genome structure and emerging evidence of an incipient sex chromosome in Populus. Genome Res. 18, 422-430. doi: 10.1101/gr.7076308

Yip, A. M., and Horvath, S. (2007). Gene network interconnectedness and the generalized topological overlap measure. BMC Bioinf. 8, 22. doi: 10.1186/1471-2105-8-22

Zhang, B., and Horvath, S. (2005). A general framework for weighted gene co-expression network analysis. Stat. Appl. Genet. Mol. Biol. 4 (1), 1128. doi: 10.2202/1544-6115.1128

Zhang, J., Li, M., Bryan, A. C., Yoo, C. G., Rottmann, W., Winkeler, K. A., et al. (2019). Overexpression of a serine hydroxymethyltransferase increases biomass production and reduces recalcitrance in the bioenergy crop Populus. Sustainable Energy Fuels 3, 195-207. doi: 10.1039/C8SE00471D

Zhang, J., Yang, Y., Zheng, K., Xie, M., Feng, K., Jawdy, S. S., et al. (2018). Genomewide association studies and expression-based quantitative trait loci analyses reveal roles of HCT 2 in caffeoylquinic acid biosynthesis and its regulation by defense-responsive transcription factors in Populus. New Phytol. 220, 502-516. doi: $10.1111 /$ nph. 15297

Zhang, J.-Y., Lee, Y.-C., Torres-Jerez, I., Wang, M., Yin, Y., Chou, W.-C., et al. (2013). Development of an integrated transcript sequence database and a gene expression atlas for gene discovery and analysis in switchgrass (Panicum virgatum L.). Plant J. 74, 160-173. doi: 10.1111/tpj.12104

Zhang, S., Chen, F., Peng, S., Ma, W., Korpelainen, H., and Li, C. (2010). Comparative physiological, ultrastructural and proteomic analyses reveal sexual differences in the responses of Populus cathayana under drought stress. Proteomics 10, 2661-2677. doi: 10.1002/pmic.200900650

Zheng, K., Wang, X., Weighill, D. A., Guo, H.-B., Xie, M., Yang, Y., et al. (2016). Characterization of DWARF14 genes in Populus. Sci. Rep. 6, 21593. doi: $10.1038 /$ srep21593

Zhou, F., and Xu, Y. (2009). RepPop: a database for repetitive elements in Populus trichocarpa. BMC Genomics 10, 14. doi: 10.1186/1471-2164-10-14

Zilberman, D., Gehring, M., Tran, R. K., Ballinger, T., and Henikoff, S. (2007). Genome-wide analysis of Arabidopsis thaliana DNA methylation uncovers an interdependence between methylation and transcription. Nat. Genet. 39, 61 doi: $10.1038 /$ ng 1929 .

Conflict of Interest Statement: The authors declare that the research was conducted in the absence of any commercial or financial relationships that could be construed as a potential conflict of interest.

Copyright $\odot 2019$ Weighill, Tschaplinski, Tuskan and Jacobson. This is an open-access article distributed under the terms of the Creative Commons Attribution License (CC BY). The use, distribution or reproduction in other forums is permitted, provided the original author(s) and the copyright owner(s) are credited and that the original publication in this journal is cited, in accordance with accepted academic practice. No use, distribution or reproduction is permitted which does not comply with these terms. 